\title{
Implicaciones ambientales de las trazas fósiles de la Formación Ixtaltepec, Carbonífero de Oaxaca, México
}

\author{
María Isabel Hernández-Ocaña, Sara A. Quiroz-Barroso
}

\begin{abstract}
María Isabel Hernández-Ocaña
marhisa@ciencias.unam.mx

Posgrado en Ciencias Biológicas, Universidad Nacional Autónoma de México, Ciudad Universitaria C.P. 04510, CDMX, México.
\end{abstract}

\section{Sara A. Quiroz-Barroso}

Museo de Paleontología, Departamento de Biología Evolutiva, Facultad de Ciencias, Universidad Nacional Autónoma de México, Ciudad Universitaria C.P. 04510, CDMX, México.

BOL. SOC. GEOL. MEX. 2018

VOL. 70 NO. 2

P. $325-350$

http://dx.doi.org/10.18268/BSGM2018v70n2a4

\section{RESUMEN}

Una de las sucesiones más completas de rocas paleozoicas de México aflora en el sur de su territorio, en el área de Santiago Ixtaltepec, en el estado de Oaxaca. En esta sucesión, las rocas carboníferas corresponden a las formaciones Santiago (TournaisianoSerpukhoviano) e Ixtaltepec (Serpu khoviano-Moscoviano). La Formación Ixtaltepec se ha dividido en ocho unidades (API-1 a API-8) caracterizadas por asociaciones fósiles de corales, trilobites, bivalvos, gasterópodos, braquiópodos, briozoos, ofiuroideos, crinoides y restos vegetales en diferentes proporciones. La unidad API-4 está compuesta de lutita y arenisca, en sus capas inferiores y medias contienen escasos fósiles corporales con bioturbación moderada. En el presente estudio se describen nueve icnotaxones para esta unidad: Cochlichnus anguineus, Cochlichnus isp., Lockeia siliquaria, Lockeia isp., Planolites isp., Protovirgularia isp., Rhizocorallium commune, y trazas asignables tentativamente a ?Gordia isp. y a ?Rhizocorallium isp., agrupados en tres icnoasociaciones. Estas icnoasociaciones corresponden a una icnofacies Cruziana e indican que el depósito ocurrió en la zona intermareal, en una planicie de marea entre mudflat y mixed flat, representando una etapa de somerización en esta área durante el Serpukhoviano-Bashkiriano.

Palabras clave: Icnoasociaciones, tafonomía, planicie de marea, Carbonífero, Fm. Ixtaltepec, México.

\section{ABSTRACT}

One of the most complete successions of paleozoic rocks is located at the SantiagoIxtaltepec area, in Oaxaca State, southern Mexico. In this succession, Carboniferous rocks are formed by the Santiago (Tournaisian-Serpukhovian) and the Ixtal tepec (Serpukhovian-Moscovian) formations. The Ixtaltepec Formation has been divided into eight units (API-1 to API-8) characterized by fossil associations of corals, trilobites, bivalves, gastropods, brachiopods, bryozoans, ophiuroids, crinoids and plant fragments, with different proportions. API-4 unit is composed of sandstone and shale, and their lower and medium layers contain few body fossils and show moderate bioturbation. The present study describes nine ichnotaxa collected in this unit: Cochlichnus anguineus, Cochlichnus isp., Lockeia siliquaria, Lockeia isp., Planolites isp., Protovirgularia isp., Rhizocorallium commune, and other traces, tentatively assignable to? Gordia isp. and ?Rhizocorallium isp., which are grouped into three associations. These ichnoassociations correspond to a Cruziana icnofacies and indicate that the deposit occurred in the intertidal zone, in a tidal plain between mudflat and mixed flat, representing a shallowing stage in this area during the Serpukhovian-Bashkirian.

Keywords: Ichnoassociations, ta phonomy, tidal flat, Carbo niferous, Ixtaltepec Formation, Mexico. 


\section{Introducción}

En el área de Santiago Ixtaltepec, Oaxaca, aflora una sucesión de depósitos marinos del Paleozoico que fue reportada por Pantoja-Alor y Robison (1967). En ella, el Carbonífero está representado por las formaciones Santiago e Ixtaltepec. Esta última se ha caracterizado por presentar una gran abundancia y riqueza de especies fósiles de diferentes grupos: trilobites (Morón y Perrilliat, 1988); bivalvos (Quiroz-Barroso, 1995; Quiroz-Barroso y Perrilliat, 1997, 1998); braquiópodos (Sour-Tovar y Quiroz-Barroso, 1989; Sour-Tovar, 1994; SourTovar y Martínez-Chacón, 2004; Torres-Martínez y Sour-Tovar, 2012, 2016a, 2016b); corales solitarios (Peña-Salinas, 2014); briozoos (Ortiz-Lozano, 1996; González-Mora, 2013; González-Mora y Sour-Tovar, 2014); ofiuroideos (Quiroz-Barroso y Sour-Tovar, 1995); crinoideos (Villanueva-Olea et al., 2011; Villanueva-Olea y Sour-Tovar, 2014) y una fructificación de pteridosperma (SilvaPineda, 1970). La sección tipo de la Formación Ixtaltepec, conocida como "Arroyo de las Pulgas", se ha dividido en 8 niveles (API-1 a API-8) que se caracterizan por su litología y por su contenido fosilífero. En la parte media (API-4) se encuentran niveles con trazas fósiles que no han sido descritas ni referidas con anterioridad, pero que aportan información valiosa para el conocimiento de los cambios ambientales que ocurrieron en el área durante el Carbonífero.

Los objetivos de este trabajo son establecer la icnotaxonomía de las trazas fósiles presentes en la unidad 4 de la Formación Ixtaltepec, así como analizar las implicaciones paleoambientales de este registro, contrastadas con la información paleoambiental de las unidades inferiores e inmediatamente superior que presentan un registro abundante de fósiles corporales y que ya han sido estudiadas previamente.

\section{Antecedentes de los estudios icnológicos}

Las trazas fósiles son estructuras sedimentarias biogénicas producto de la actividad y comportamiento de los organismos, que se han preservado

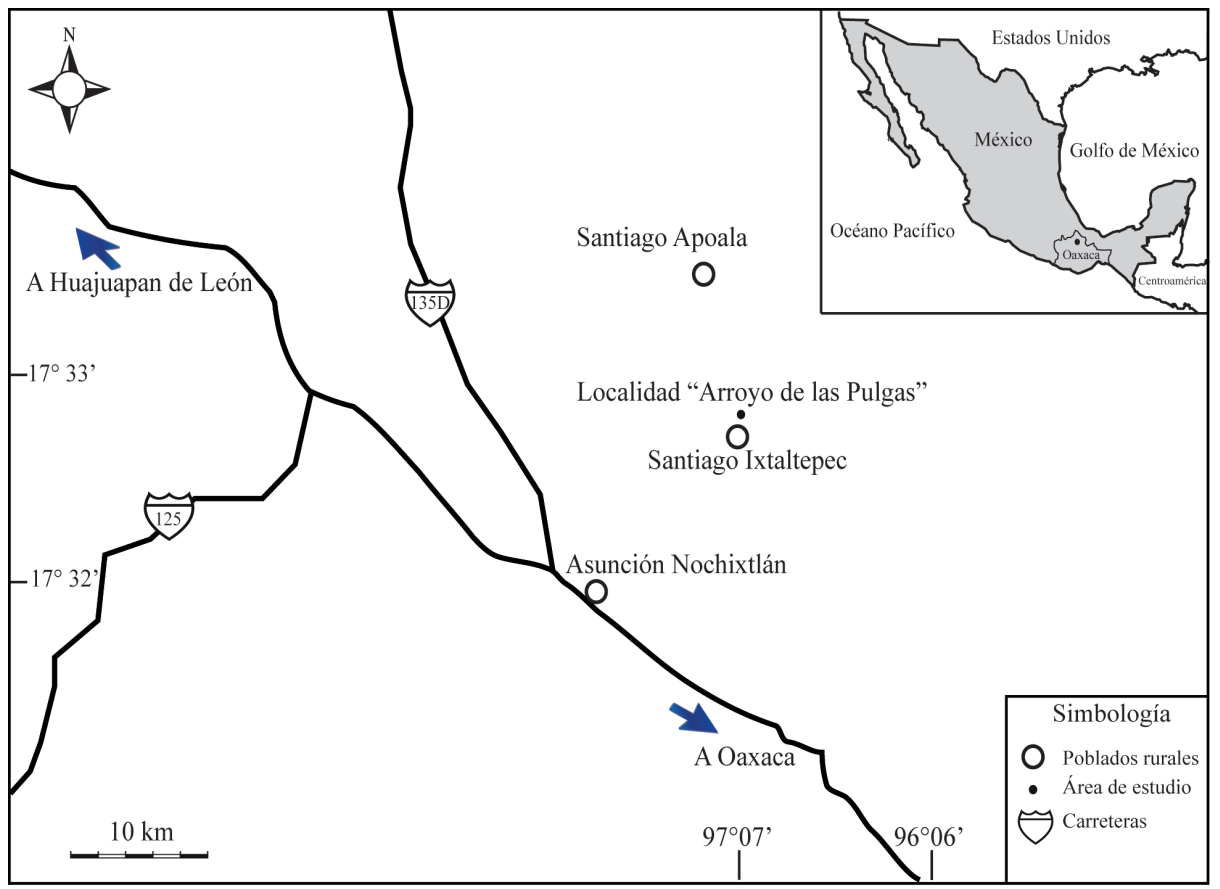

Figura 1 Ubicación geográfica de Santiago Ixtaltepec, en donde aflora la sección tipo "Arroyo de las Pulgas", al noreste de la cabecera municipal Asunción Nochixtlán, en el estado de Oaxaca, México. 
en el registro estratigráfico y que se pueden reconocer por su doble naturaleza, tanto como objetos biológicos como sedimentológicos (Pemberton et al., 2000), por lo que su estudio resulta ser una herramienta importante para la Paleontología y en el análisis de ambientes sedimentarios. Las asociaciones de las trazas fósiles de invertebrados se consideran como la respuesta de los organismos bentónicos a un conjunto determinado de parámetros ambientales, tales como energía, tasa de sedimentación, salinidad del medio, niveles de oxígeno, interacción con otros organismos y suministro de alimento (Ekdale, 1985; Bromley, 1996; Pemberton et al., 2000; McIlroy, 2004; Buatois y Mángano, 2008), por lo que el estudio de las asociaciones de las trazas fósiles resulta fundamental para obtener información paleoambiental (Buatois y Mángano, 2008).

Una icnofacies consiste en una asociación de trazas fósiles que se repite en el tiempo geológico cuando las condiciones ambientales también lo hacen, por lo que tiene un carácter recurrente; al estar asociada a determinados parámetros ambientales, permite el análisis paleoambiental. También se trata de agrupaciones de estructuras biogénicas que reflejan las respuestas de los animales (etología) a las condiciones paleoambientales (MacEachern et al., 2007, 2012; Buatois y Mángano, 2011). Las icnofacies arquetípicas o seilacherianas corresponden a asociaciones de trazas fósiles recurrentes en diferentes depósitos a lo largo del Fanerozoico y en diferentes lugares de la geografía mundial (de Gibert y Martinell, 1998). Las icnofacies constituyen asociaciones que engloban los rasgos comunes de una serie de icnofaunas particulares, por lo que si bien cada icnofacies es denominada con un icnogénero típico, su definición se basa en una serie de características diagnósticas y no en la presencia del icnogénero epónimo (de Gibert y Martinell, 1998; Buatois y Mángano, 2008). Originalmente se consideró que las icnofacies marinas son indicadoras de batimetría (Seilacher, 1967), pero esto no es necesariamente cierto (Crimes, 1977; Seilacher, 1978; Pemberton y Frey, 1984; Buatois y Mángano, 2008). Los principales controles en la distribución de icnofacies incluyen tipo de sustrato, energía, contenido de nutrientes, oxigenación, salinidad, tasas de sedimentación y erosión, siendo la batimetría un control de segundo orden (de Gibert y Martinell, 1998; Buatois y Mángano, 2008). Así que, se deben considerar todos los datos posibles tanto sedimentológicos como de los icnogéneros más representativos, pues la gama ambiental de algunas trazas fósiles puede cambiar a través del tiempo, por ejemplo, la migración de las trazas en ambientes marinos someros a ambientes profundos y viceversa (Crimes y Fedonkin, 1994; Olivero y Gaillard, 1996; Olivero, 2003).

\section{Ubicación del área de estudio y estratigrafía}

El material estudiado se recolectó en la sección tipo de la Formación Ixtaltepec en el "Arroyo

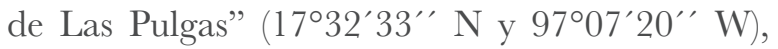
aproximadamente a $16 \mathrm{~km}$ al noreste del poblado de Asunción Nochixtlán y a 500 m al norte del poblado de Santiago Ixtaltepec, Oaxaca (Figura $1)$.

Las rocas más antiguas que afloran en el área son precámbricas, con edades de 900 a 1100 Ma (Fries et al., 1962; Solari et al., 2003). Dichas rocas corresponden al Complejo Oaxaqueño, compuesto de pegmatita, esquisto, paragneis y ortogneis. Sobre el complejo proterozoico descansan, en inconformidad, sedimentos del Cámbrico-Ordovícico correspondientes a la Formación Tiñú (PantojaAlor, 1970; Sour-Tovar y Buitrón-Sánchez, 1987). Sobreyaciendo a estas rocas, en inconformidad, se encuentra la Formación Santiago, unidad informal por homonimia de edad misisípica (Tournaisiano-Serpukhoviano; Quiroz-Barroso et al., 2000; Navarro-Santillán et al., 2002; CastilloEspinoza, 2013), que tiene un espesor de $165 \mathrm{~m}$ y está dividido en dos miembros: el inferior se caracteriza por la presencia de rocas calcáreas. Mientras que en el superior predomina la lutita con intercalaciones de limolita, arenisca y caliza (Figura 2). 
La Formación Santiago subyace transicionalmente a la Formación Ixtaltepec de edad Serpukhoviano-Moscoviano, correspondiente al Misisípico Tardío-Pensilvánico Medio (SourTovar y Martínez-Chacón, 2004; Torres-Martínez y Sour-Tovar, 2012, 2016a, 2016b; VillanuevaOlea y Sour-Tovar, 2014). La sección tipo de la
Formación Ixtaltepec presenta un espesor de $430 \mathrm{~m}$, en su base inicia con limolita, seguida de intercalaciones de lutita y calcarenita; por encima se depositan capas delgadas de calcarenita ligeramente arcillosa con bancos gruesos de lutita arenosa de colores grisáceos e intercalaciones de arenisca de grano fino. A partir de la parte media

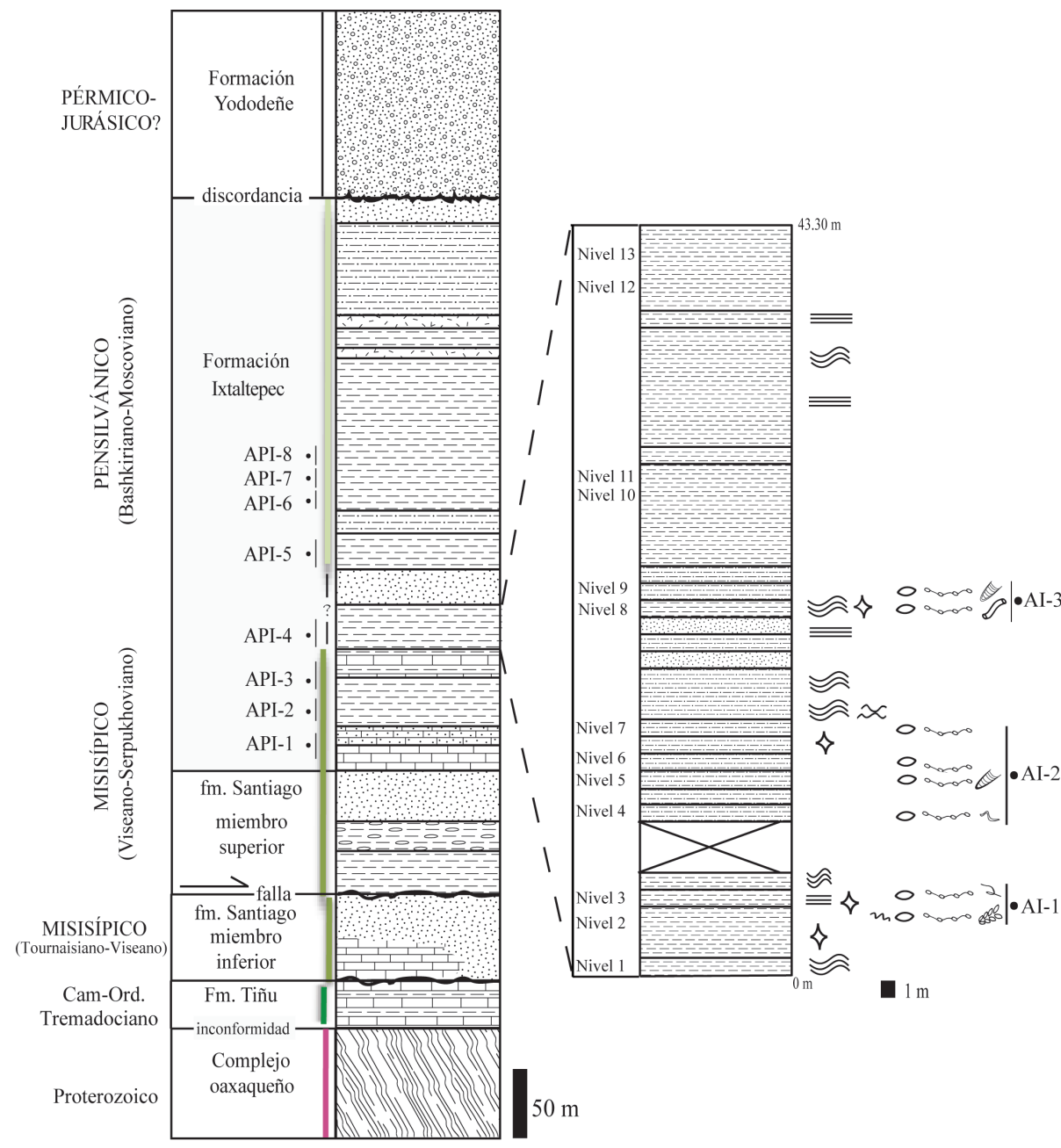

\begin{tabular}{|c|c|c|c|c|c|}
\hline \multicolumn{3}{|c|}{ Litología } & Estructuras sedimentarias & \multicolumn{2}{|l|}{ Contenido icnofosílifero } \\
\hline 0 & $==-$ & 4.m. & $\equiv$ Laminación paralela & Cochlichnus anguineus & $m$ \\
\hline Conglomerado & Lutita & Rocas & $\approx$ Rinples de corriente & Cochlichnus isp. & 2 \\
\hline 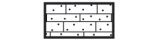 & 目 & Metamórficas & S кipples de commente & $\begin{array}{l}\text { Lockeia siliquaria } \\
\text { Lockeia isp. }\end{array}$ & 0 \\
\hline Calcarenita & Lutita arenosa & 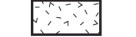 & $\approx$ Estratificación tipo flaser & Planolites isp. & $\infty$ \\
\hline a & 官三春 & Rocas & $\diamond$ Ripples de interferencia & Protovirgularia isp. & $\operatorname{sos}_{0}^{\infty 00}$ \\
\hline Arenisca & Lutita con & Intrusivas & $\Longrightarrow \quad \sim$ & ?Gordia isp. & 2 \\
\hline 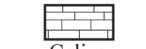 & concreciones & & Falla de ángulo bajo Zona plegada & $\begin{array}{l}\text { Rhizocorallium commune } \\
\text { Rhizocorallium isp. }\end{array}$ & $e$ बाँ \\
\hline Calıza & & $\begin{array}{l}\text { Sección } \\
\text { cubierta }\end{array}$ & & & \\
\hline
\end{tabular}

Figura 2 (A) Columna estratigráfica de la sección tipo “Arroyo de las Pulgas", el acrónimo API significa Arroyo de las Pulgas (Modificada de Villanueva et al., 2011, con base en los trabajos de Quiroz-Barroso y Perrilliat, 1998 y Navarro-Santillán et al., 2002). (B) Sección estratigráfica de la unidad API-4 de la Formación Ixtaltepec en la que se señalan los niveles fosilíferos. 
se presentan estratos de limolita y arenisca micácea de grano fino, bancos gruesos de lutita, lutita arenosa y arenisca de grano fino. En el último tercio de la sección se observan intrusiones de diorita a manera de estratos intercalados con lutita arenosa y lutita. Informalmente, para el análisis paleontológico de la formación se han marcado ocho niveles de estudio (API-1 a API-8). Cada nivel presenta características litológicas particulares y contiene una asociación fósil distintiva en todo su espesor (Ortiz-Lozano, 1996; Quiroz-Barroso y Perrilliat, 1997, 1998; Torres-Martínez y Sour-Tovar, 2012, 2016a, 2016b).

Las unidades API-1 y API-2 se componen principalmente de caliza y calcarenita. Se ha referido la presencia de corales rugosos, braquiópodos, briozoos y crinoideos (Sour-Tovar, 1994; VillanuevaOlea et al., 2011), sugiriendo que el depósito se formó en un ambiente somero, de aguas claras, con un sustrato arenoso y con precipitaciones de carbonatos, alta productividad, un nivel energético medio y un aporte de terrígenos moderado (PeñaSalinas, 2014; Torres-Martínez, 2014). Sus características tafonómicas (concentraciones in situ y parautóctonas de origen biogénico) indican que la parte superior de la unidad API-1 y la parte inferior de la API-2 se depositaron en la plataforma exterior, en condiciones someras (Hernández-Ocaña, 2016). Además, recientemente se ha reportado la presencia de algas calcáreas, foraminíferos y gasterópodos (González-Mora, 2017), resaltando las condiciones someras y la influencia arrecifal (Figura 3A-C).

La unidad API-3 está compuesta de lutita de tonos abigarrados. La concentración fósil es in situ y parautóctona de origen biogénico (HernándezOcaña, 2016), se encuentra en un solo nivel, en la parte media de esta unidad, de donde se ha referido la presencia de briozoos, bivalvos y crinoideos (Quiroz-Barroso y Perrilliat, 1997, 1998; Villanueva-Olea, 2011). Torres-Martínez (2014), estudia los abundantes braquiópodos (Figura 3D) y menciona un ambiente de depósito de fondo lodoso, baja energía y alta productividad, con un aporte continuo de terrígenos, principalmente arcillas. De acuerdo a las asociaciones faunísticas que se encuentran se ha relacionado al depósito con un ambiente submareal. Algunos de esos braquiópodos presentan adaptaciones que les permitían vivir sobre el sustrato sin hundirse o ser sepultados, por ejemplo, las conchas de Echinoconchella elegans o Echinoconchus zapoteco poseían una geometría plano-convexa y eran muy ligeras. Otras formas, como las especies de Inflatia y de Marginovatia, poseían una concha con la valva ventral fuertemente convexa que les permitía vivir ligeramente enterradas pero manteniendo la valva dorsal y el lofóforo sobre la interfase sedimento-agua (Torres-Martínez y Sour-Tovar, 2012, 2016a, 2016b).

La unidad API-4 consiste en lutita, lutita arenosa y arenisca con 13 niveles fosilíferos: los inferiores y medios (niveles 1-9) contienen las trazas fósiles objeto de este estudio (Figura 2), un fragmento de planta y escasos bivalvos. En la parte superior de la unidad API-4 (niveles 10 - 13) se encuentran fósiles de trilobites, bivalvos, braquiópodos, crinoideos y briozoos que se presentan en acumulaciones ex situ y alóctonas de origen sedimentológico. También hay presencia de pequeñas concentraciones in situ y parautóctonas de origen biogénico de crinoideos pequeños, fragmentos de trilobites, y hiolítidos (Hernández-Ocaña, 2016; Figura 3E y F).

La unidad API-5 consiste de arenisca y lutita arenosa con escasa presencia de ripples. Se presentan fósiles de briozoos y de braquiópodos (Figura 3G), que se encuentran en concentraciones in situ y parautóctonas de origen biogénico (HernándezOcaña, 2016). Estos organismos son abundantes en aguas marinas poco profundas. Entre los braquiópodos se encuentran: Echinoconchella elegans, Orbiculoidea capuliformis, Neospirifer dunbari, Neospirifer pantojai, Neospirifer amplia y Septospirifer mazateca que se han asociado a un ambiente submareal somero (Torres-Martínez y Sour-Tovar, 2012, 2016a, 2016b), por lo que se ha considerado que 

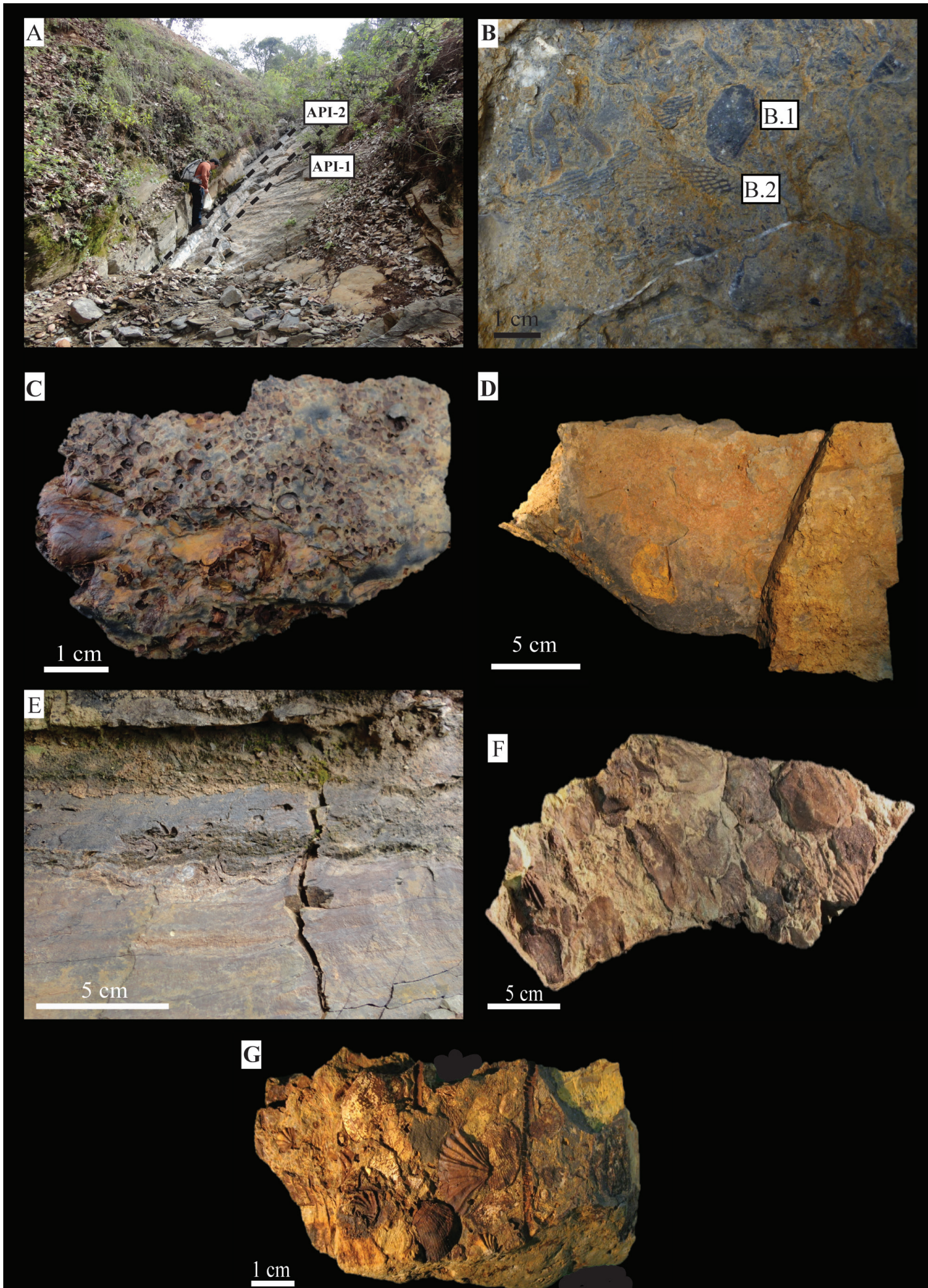

Figura 3 (A) Contacto entre las unidades API-1y API-2. (B) Concentraciones de braquiópodos (B.1) y briozoos (B.2) en la unidad API-1. (C) Concentraciones de crinoideos y braquiópodos en la unidad API-2, FCMP TF 1. (D) Concentración de braquiópodos en la unidad API3. (E) Corte transversal con lentes de restos de organismos en la unidad API-4. (F) Acumulación de organismos mal preservados como braquiópodos y bivalvos en la unidad API-4, FCMP TF 2. (G) Concentración de braquiópodos y crinoideos en la base de la unidad API-5, FCMP TF 3. 
su depósito ocurrió en un ambiente de baja energía, con fondo lodoso, bien iluminado y de aguas cálidas, de plataforma externa.

Las unidades superiores (API-6 a API-8) presentan la mayor diversidad y abundancia de fósiles corporales y se han relacionado con un depósito en zonas periarrecifales (Quiroz-Barroso y Perrilliat 1997, 1998; Torres-Martínez, 2014; Torres-Martínez y Sour-Tovar, 2012, 2016a, 2016b).

La sucesión paleozoica termina con la Formación Yododeñe, compuesta por un conglomerado con clastos calcáreos de edad pérmico-jurásica; sobre esta formación se observan rocas calcáreas de edad cretácica (Pantoja-Alor, 1970).

\section{Metodología}

Se identificaron y describieron los icnotaxones presentes en la parte inferior y media de la unidad API-4 de la Formación Ixtaltepec. Se revisó la información etológica que representan estos icnotaxones y se establecieron tres icnoasociaciones. A partir de lo anterior, se reconoció la icnofacies que representan y se interpretó el paleoambiente en el que ocurrió el depósito, comparándolo con los ambientes reconocidos previamente para las unidades inferiores (API-1, API-2, API-3) y la superior inmediata (API-5).

\section{Resultados}

\subsection{IGNOTAXONOMÍA}

El material proviene de diferentes niveles inferiores y medios de la unidad API-4, de edad Serpukhoviano-Bashkiriano. Los ejemplares estudiados se clasificaron de acuerdo a sus icnotaxobases (en el sentido de Bromley, 1996) y están depositados en el Museo de Paleontología de la Facultad de Ciencias, Universidad Nacional Autónoma de México, con el acrónimo FGMP. La abundancia relativa de cada icnotaxón en la unidad API-4 es: muy escaso = 1 espécimen; escaso $=2$ a 10 especímenes; común $=11$ a 20 especímenes; abundante $=>$ de 20 especímenes.

\section{Icnogénero Cochlichnus Hitchcock, 1858}

Icnoespecie tipo. Cochlichnus anguineus Hitchcock, 1858, Jurásico, Valle de Connecticut, E.U.A.

Diagnosis. Excavaciones de interfase o pistas continuas y regularmente serpenteantes, que se asemejan a una onda sinuosa o a una espiral completa. La curvatura que presenta puede disminuir gradualmente de amplitud (enmendada por Keighley y Pickerill, 1997).

\section{Cochlichnus anguineus Hitchcock, 1858} (Figura 4A)

Sinonimia. Para una revisión completa, ver Stanley y Pickerill (1998).

Diagnosis. La misma del icnogénero.

Material analizado. FCMP 1185. Muy escaso. Descripción. Traza sinuosa y curveada que se adelgaza al final de las curvas; paredes con un margen hundido tenuemente. La longitud de la traza es de aproximadamente $4 \mathrm{~cm}$; la amplitud de las curvas es de $1 \mathrm{~cm}$ de ancho. Se conserva en hiporrelieve.

Discusión. La traza estudiada se asigna a Cochlichnus anguineus ya que la forma que presenta la ondulación de las curvas y su extensa amplitud, de aproximadamente $1 \mathrm{~cm}$, es similar a la reportada por Stanley y Pickerill (1998).

El icnogénero Cochlichnus se ha descrito como formas sinuosas y vermiformes que constituyen un ejemplo típico de movimiento ondulado (Seilacher, 2007). Cochlichnus se ha encontrado en asociación con los icnogéneros Planolites, Lockeia, Protovirgularia, Helminthoidichnites (Głuszek, 1995; Buatois et al., 1997; Balistieri et al., 2002; Buatois y Mángano, 2007), algunos de los cuales se encuentran en los mismos estratos en donde se encontró este ejemplar en la unidad API-4 de la Formación Ixtaltepec. 
Localidad. Arroyo de las Pulgas, nivel 3 (a 4.5 $\mathrm{m}$ de la base en la unidad API-4), Formación Ixtaltepec.

\section{Cochlichnus isp.}

(Figura 4B.1)

Material analizado. FCMP 1186. Muy escaso. Descripción. Traza simple y curveada, de 2.5 cm de longitud, la amplitud de cada curva es de aproximadamente $5 \mathrm{~mm}$. La traza se conserva en epirrelieve.

Discusión. Cochlichnus isp., es una traza sinuosa y se asigna como isp. por el tamaño tan pequeño con respecto al de la especie $C$. anguineus y por tratarse de trazas preservadas en epirrelieve.

Localidad. Arroyo de las Pulgas, nivel 4 (a 9 m de la base en la unidad API-4), Formación Ixtaltepec.

\section{Icnogénero Gordia Emmons, 1844}

Icnoespecie tipo. Gordia marina Emmons, 1844, Cámbrico Superior, Taconic System, Nueva York, E.U.A.

Diagnosis. Senderos no ramificados, predominantemente horizontales, o madrigueras serpenteadas regularmente con bucles (loops), con una marcada tendencia a entrecruzarse; sin estructuras de relleno (diagnosis enmendada por Pickerill y Peel, 1991; Stanley y Pickerill, 1998).

\section{?Gordia isp.}

(Figura 4C-E)

Material analizado. FCMP 1187, FGMP 1188 y FCMP 1189. Escaso.

Descripción. Trazas que asemejan meandros, muy angostas. Una característica distintiva es que se entrecruzan, formando bucles. Diámetro de 10 mm aproximadamente, el cual es complicado de medir por la preservación de las trazas. Preservadas en hiporrelieves sobre el plano de estratificación.

Discusión. Estas trazas se asignan con duda al icnogénero de Gordia, ya que una característica distintiva del icnogénero es el entrecruzamiento de la traza, lo que se puede observar únicamente en una de las trazas estudiadas de la Formación Ixtaltepec. Difiere de Helminthopsis ya que éste consiste en una traza relativamente simple, representando también un sendero irregular y sinuoso sin ramificaciones, pero sin el entrecruzamiento (Han y Pickerill, 1995). Otro icnogénero comparable con Gordia es Helminthoidichnites, estas son trazas pequeñas y horizontales, rectas o curveadas, pero raramente circulares y en algunos casos pueden sobreponerse con otras trazas (Buatois et al., 1998). Localidad. Arroyo de las Pulgas, nivel 3 (a 4.5 $m$ de la base en la unidad API-4), Formación Ixtaltepec.

\section{Icnogénero Lockeia James, 1879}

Sinonimia. (= Pelecypodichnus Seilacher, 1953). Icnoespecie tipo. Lockeia siliquaria James, 1879, Ordovícico Superior, Ohio, E.U.A.

Diagnosis. Traza con simetría bilateral, alargada, con forma de almendra, forma de corazón, forma de mancuerna o rara vez triangular, con el margen liso; predominantemente preservado en formas aisladas o en montículos alineados en hiporelieves; segmentos individuales distintos comúnmente con una cresta mediana. Pueden estar presentes formas onduladas (enmendado por Schlirf et al., 2001).

\section{Lockeia siliquaria James 1879}

(Figura 5A)

Sinonimia. Para una revisión completa, ver Schlirf et al. (2001).

Diagnosis. Estructuras delgadas, de alargadas a anchas, generalmente con un alto relieve en forma de almendra, crestas lisas, fuertemente arqueadas en las terminaciones casi obtusas. Ocasionalmente se presentan spreiten verticales (enmendado por Schlirf et al., 2001).

Material analizado. FGMP 1190, FGMP 1191, FCMP 1192. Abundante.

Descripción. Formas de ovaladas a circulares ligeramente elongadas, con las paredes lisas y sin 


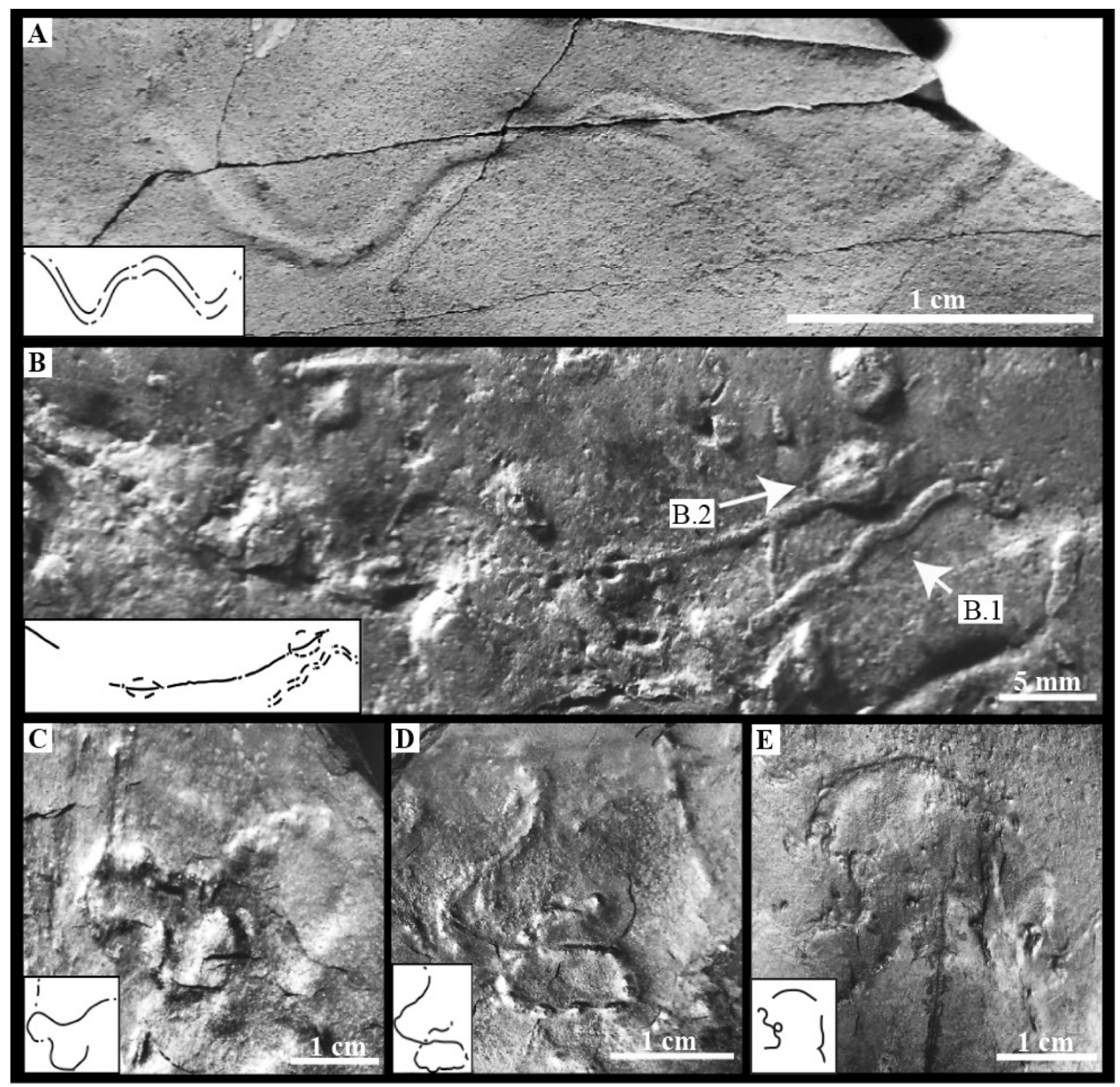

Figura 4 (A) Cochlichnus anguineus, FCMP 1185. (B.1) Cochlichnus isp., FCMP 1186. (B.2) Lockeia isp., FCMP 1193. (C - E) ?Gordia isp.: C, FCMP 1189; D, FCMP 1187; E, FCMP 1188. La posición de las pistas en las fotografías se representa en los recuadros.

ornamentación visible. Las terminaciones son ligeramente obtusas y en un extremo presentan una cresta tenue. Las trazas están conservadas en hiporrelieves, en posición horizontal al plano de estratificación, sin una dirección definida. El largo mayor es de $1 \mathrm{~cm}$, con $5 \mathrm{~mm}$ de ancho.

Discusión. El icnogénero Lockeia ha sido objeto de diversas interpretaciones, describiéndose especies que dada su preservación, han sido reconsideradas como formas intermedias (Schlirf et al., 2001). Para poder distinguir a las icnoespecies se utilizan características como la forma típica ovalada o de almendra, la terminación de las puntas, el tamaño y su preservación. El material descrito para la Formación Ixtaltepec se asigna a Lockeia siliquaria ya que las formas son pequeñas y ovaladas, alargadas y lisas en sus paredes, en algunos casos se encuentran agrupadas. Estructuras similares han sido reportadas por Mángano et al. (1998) para el Carbonífero Superior del este de Kansas, en donde se reconoce que L. siliquaria no está conectada con otras trazas, pero sí puede estar conectada por medio de ejes en epirrelieves, patrón que también muestran las formas estudiadas. Otra forma similar a $L$. siliquaria, es $L$. amigdaloides (Seilacher, 1953), de la que únicamente difiere en sus dimensiones, concluyéndose que sólo es una variante de $L$. siliquaria. Por otra parte, 
se puede comparar a $L$. siliquaria con Lockeia ornata (Bandel, 1967), sin embargo, esta última presenta una cresta media longitudinal, en algunos casos tiene la impresión de las comisuras de las valvas del organismo productor, y está conectada a trazas en forma de chevrón, consideradas estructuras de locomoción (Repichnia; Mángano et al., 1998).

Localidad. Arroyo de las Pulgas, niveles 2 y 3 (a 4 y 4.5 m de la base en la unidad API-4), nivel 4 (a $9 \mathrm{~m}$ de la base en la unidad API-4), niveles 5, 6 y 7 (a 10, 12.5 y $15 \mathrm{~m}$ de la base en la unidad API-4, respectivamente), nivel 8 (a $20 \mathrm{~m}$ de la base en la unidad API-4), nivel 9 (a $22 \mathrm{~m}$ de la base en la unidad API-4), Formación Ixtaltepec.

\section{Lockeia isp.}

(Figuras 4B.2 y 5B)

Material analizado. FGMP 1193, FGMP 1194.

Abundante.

Descripción. Trazas con formas ovaladas y con una ligera cresta longitudinal. Las puntas presentan un ángulo muy agudo y las paredes, dada su preservación, son irregulares. Tienen un patrón definido en donde se observa que las trazas están conectadas unas con otras. Las medidas que poseen van de $2.5 \mathrm{~cm}$ de largo a $1 \mathrm{~mm}$ de ancho. Se encuentran conservadas tanto en epirrelieves como hiporrelieves, en posición horizontal al plano de estratificación.

Discusión. Difiere de Lockeia siliquaria por ser formas más grandes, y a pesar de representar trazas de descanso o Cubichnia, están relacionadas con una estructura de movimiento o desplazamiento, muy similares a las que asignaron Seilacher y Seilacher (1994) como Lockeia serialis, aunque $L$. serialis se considera como nomen oblitum (Schlirf et al., 2001). Esta traza que se describe podría asignarse también con duda a Lockeia siliquaria, debido a que, como se explicó anteriormente, las icnoespecies del género Lockeia se han considerado como formas intermedias.

Localidad. Arroyo de las Pulgas, niveles 2 y 3 (a 4 y $4.5 \mathrm{~m}$ de la base en la unidad API-4), nivel 4 (a $9 \mathrm{~m}$ de la base en la unidad API-4), niveles 5, 6 y 7 (a 10, 12.5 y $15 \mathrm{~m}$ de la base en la unidad API-4, respectivamente), nivel 8 (a $20 \mathrm{~m}$ de la base en la unidad API-4), nivel 9 (a $22 \mathrm{~m}$ de la base en la unidad API-4), Formación Ixtaltepec.

\section{Icnogénero Planolites Nicholson, 1873}

Icnoespecie tipo. Planolites beverleyensis Billings, 1862, Silúrico, Canadá.

Diagnosis. Tubos cilíndricos de diámetro constante a lo largo de un mismo ejemplar, de paredes lisas, con diferente grado de curvatura o rectos, no ramificados y dispuestos en cualquier posición en el espacio (Pemberton y Frey, 1982).

\section{Planolites isp.}

(Figura 5C)

Material analizado. FGMP 1195. Abundante.

Descripción. Trazas de tubos cilíndricos y entrecruzados, sin ramificaciones. Se encuentran formando estructuras de excavación que van perforando el sustrato y se conservan en hiporrelieves negativos. Los tubos tienen una longitud de 1 a 5 $\mathrm{cm}$ y un ancho de $1 \mathrm{~cm}$. Las paredes de las trazas están pobremente preservadas y presentan una ornamentación irregular. El sedimento que rellena las trazas es arena, diferente al que las contiene.

Discusión. Debido a que el material no presenta una buena preservación, sólo se ha asignado a nivel de icnogénero. Estas trazas se pueden diferenciar de Planolites montanus (Richter, 1937) que es una traza más pequeña y más curveada, generalmente sin estructuras de revestimiento; otra icnoespecie comparable es Planolites beverleyensis (Billings, 1862) pero esta última presenta estructuras más continuas (Fillion y Pickerill, 1990). Las descripciones que se han realizado acerca de este icnogénero refieren que se debe a la actividad de un organismo detritívoro que excava irregularmente en un sustrato, por lo común arenoso o fangoso, en busca de nutrientes (Garcia-Ramos, 1984). Varios autores han evaluado la taxonomía de Planolites (Pemberton y Frey, 1982; Fillion y Pickerill, 1990; Keighley y Pickerill, 1995). Algunos de estos 

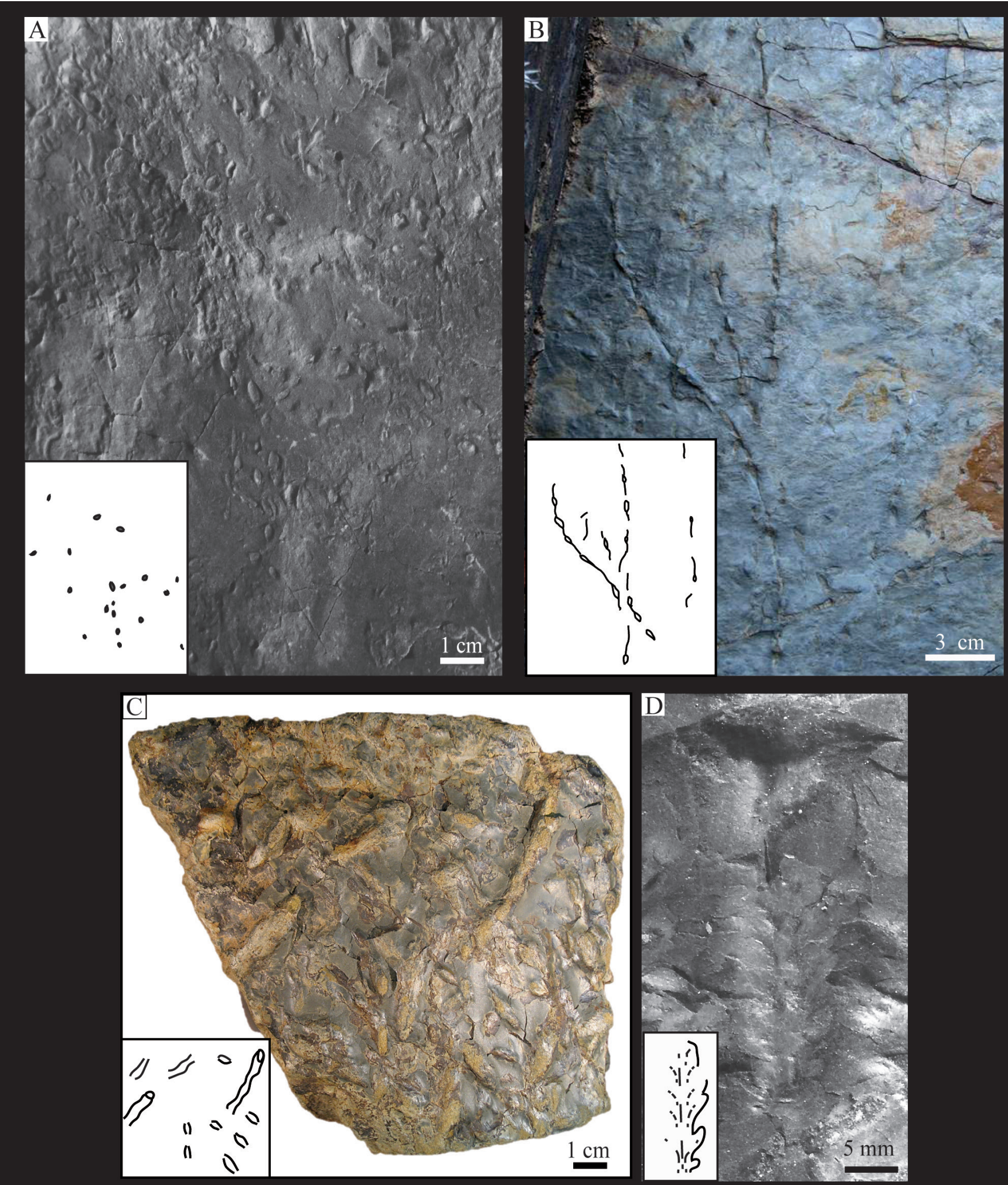

Figura 5 (A) Lockeia siliquaria: numerosos ejemplares aislados o formando grupos pequeños, FCMP 1192 . (B) Lockeia isp., fotografía de campo, molde en silicón FCMP 1194. (C) Planolites isp., FCMP 1 195. (D) Protovirgularia isp., FCMP 1196, vista de la superficie horizontal. La posición de las pistas en las fotografías se representa en los recuadros. 
autores mencionan que morfológicamente es similar al icnogénero Palaeophycus, llegando a la conclusión que Planolites representa una estructura de alimentación sin forro que se rellenó activamente y que el relleno es diferente al sustrato que contiene a la traza, mientras Palaeophycus representa una excavación que fue forrada de una pared con un rayado paralelo, tal como lo señala Häntzschel (1975).

Localidad. Arroyo de las Pulgas, nivel 8 (a 20 $\mathrm{m}$ de la base en la unidad API-4), Formación Ixtaltepec.

\section{Icnogénero Protovirgularia M'Coy, 1850}

Sinonimia. Para una revisión completa, ver Seilacher y Seilacher (1994).

Icnoespecie tipo. Protovirgularia dichotoma M'Coy, 1850, Silúrico Inferior, Southern Uplands, Escocia.

Diagnosis. Trazas pequeñas en forma de quilla, con una línea media y apéndices laterales en forma de cuña alternándose en ambos lados.

\section{Protovirgularia isp.}

(Figura 5D)

Material analizado. FCMP 1196. Muy escaso. Descripción. Pista horizontal preservada en hiporrelieve tenue, con un patrón muy característico formado por la repetición de una estructura en espiga (chevrón), las líneas que dan lugar a la forma de chevrón se disponen en V. Presenta un ligero eje en medio de la estructura. El largo es de $1.5 \mathrm{~cm}$, el ancho máximo es de $4 \mathrm{~mm}$, esto es debido a que las líneas van reduciendo su tamaño. Discusión. La traza descrita para la Formación Ixtaltepec se preserva en hiporrelieve positivo y negativo. Se encuentra dentro del sustrato y muestra similitudes con la icnoespecie Protovirgularia dichotoma (M'Goy, 1850), pero la forma en chevrón no está muy marcada debido posiblemente a los procesos tafonómicos a los que las trazas fueron expuestas, lo que impide asignarla icnoespecíficamente. Además se encuentra aislada y no asociada al icnogénero Lockeia como generalmente se ha referido.

El icnogénero Protovirgularia ha sido referido y discutido por diversos autores. Previamente ha sido reconocido como un octocoral, como algas marinas (M'Coy, 1850), como graptolito (Richter, 1853), o como una estructura hecha por cangrejos (Gümbel, 1879) y artrópodos (Richter, 1941; Volk, 1961). Tiempo después Häntzschel (1975) consideró a Protovirgularia como un icnofósil. En diversos trabajos se ha demostrado que el icnogénero Protovirgularia y su forma es resultado de la actividad del pie bífido de bivalvos protobranquios con hábitos excavadores y rastreros en sustratos suaves (Seilacher y Seilacher, 1994; de Gibert y Domènech, 2008). También se ha interpretado que Protovirgularia es una traza posiblemente realizada por bivalvos nuculoideos (de Gibert y Domènech, 2008).

Localidad. Arroyo de las Pulgas, nivel 3 (a 4.5 $\mathrm{m}$ de la base en la unidad API-4), Formación Ixtaltepec.

\section{Icnogénero Rhizocorallium Zenker, 1836}

Icnoespecie tipo. Rhizocorallium jenense Zenker, 1836. Triásico Inferior-Medio, Turingia, Alemania.

Diagnosis. Traza de madriguera horizontal a oblicua, spreiten en forma de U (enmendado por Knaust, 2013).

\section{Rhizocorallium commune Schmid, 1876} (Figura 6A, B)

Sinonimia. Para una revisión completa ver Knaust (2013).

Diagnosis. Excavaciones no ramificadas o raramente ramificadas, con orientación preferentemente subhorizontal. Las excavaciones son alargadas, en forma de línea, rectas o sinuosas, y pueden tener estrías longitudinales subparalelas en la pared. Comúnmente están asociadas con pellets fecales (Coprolus isp.), que pueden estar 
dentro del spreiten y de los tubos (enmendado por Knaust, 2013).

Material analizado. FGMP 1197. Escaso.

Descripción. Traza en forma cilíndrica y linear en posición horizontal, con presencia de un tenue revestimiento y sin ramificaciones. El spreiten se encuentra ligeramente espaciado, con líneas horizontales o segmentos con un sedimento arenoso, donde se observa el efecto de un movimiento protusivo. Su longitud es de $10 \mathrm{~cm}$ aproximadamente y tiene un ancho de $2.5 \mathrm{~cm}$ aproximadamente. Se preserva en relieve completo.

Discusión. Se han reconocido dos especies del icnogénero Rhizocorallium, considerando como diferencias la forma en que se conservan en el plano de estratificación: Rhizocorallium jenense (Zenker, 1836) y Rhizocorallium commune (Schmid, 1876). Las trazas encontradas en la Formación Ixtaltepec se asignan a Rhizocorallium commune por su posición horizontal y el revestimiento
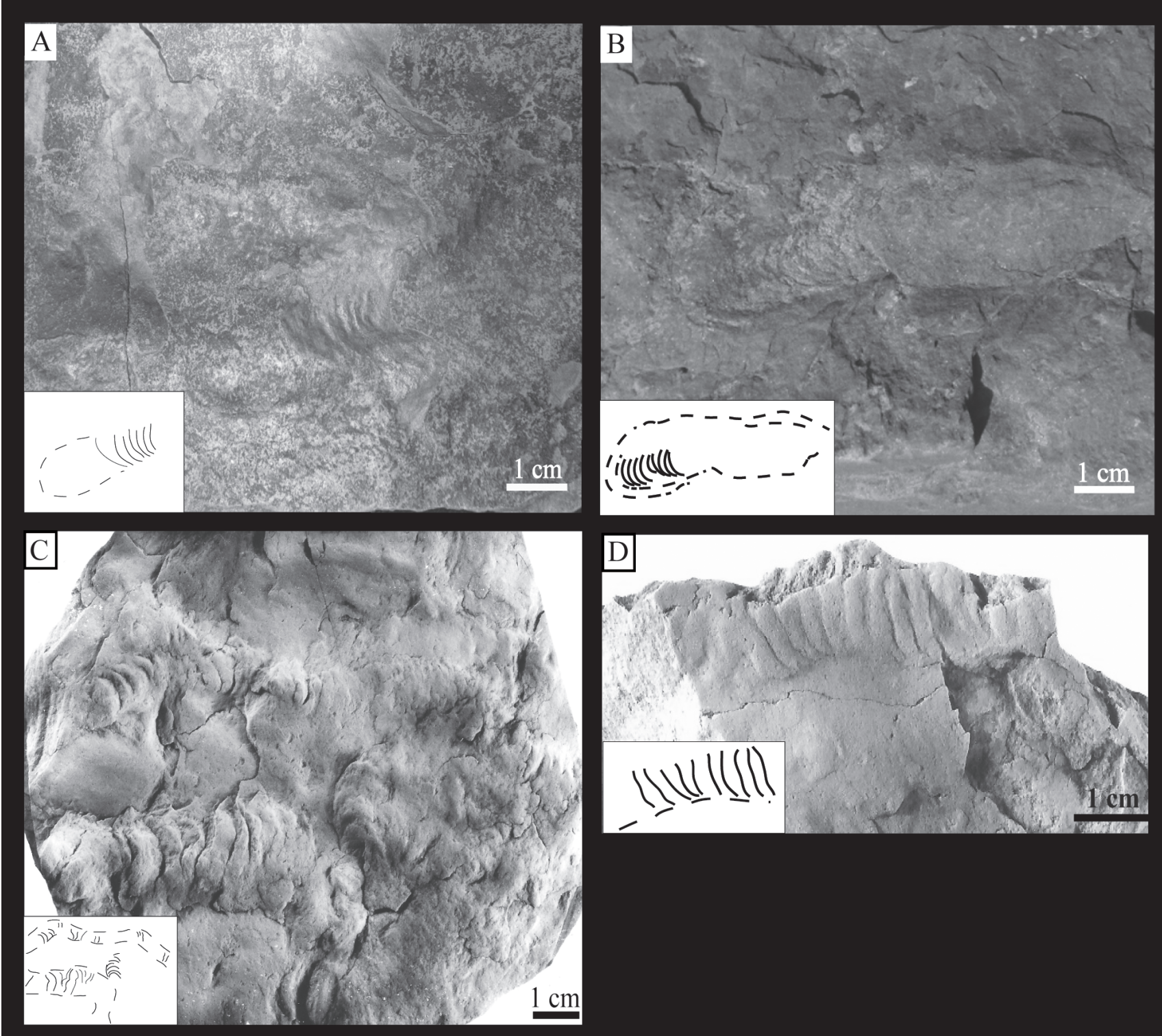

Figura 6 (A, B) Rhizocorallium commune: A, FCMP 1197; B, fotografía de campo. (C, D) ?Rhizocoralium isp.: C, FCMP 1198 ; D, FCMP 1199. 
típico alrededor de la traza (Knaust, 2013), no se observan arañazos en la pared. Una característica distinguible de la icnoespecie es la presencia de pellets fecales, no observados en la traza descrita debido posiblemente a la mala preservación del material estudiado. Un icnogénero similar a las trazas descritas es Beaconites, que si bien presenta una orientación horizontal en el sustrato, se caracteriza por la forma de los surcos o estrías y su forma de U alargada, además de carecer de un revestimiento tubular típico de Rhizocorallium. Su distribución puede estar influenciada por condiciones ambientales específicas (como el tipo de sustrato, energía, oxígeno, nutrientes y salinidad) y por lo tanto su morfología y el tamaño pueden variar (Knaust, 2013).

Localidad. Arroyo de las Pulgas, nivel 9 (a 22 $m$ de la base en la unidad API-4), Formación Ixtaltepec.

\section{?Rhizocorallium isp.}

(Figura 6C, D)

\section{Material analizado. FGMP 1198 y FGMP} 1199. Escaso.

Descripción. Trazas en forma de U con un spreiten con estrías o surcos bien definidos y una ligera imbricación, sin presencia de un revestimiento. Posee 10 surcos que miden de 2 a $3 \mathrm{~mm}$ de ancho cada uno. Se preservan en epirrelieves positivos, siguiendo el plano de estratificación.

Discusión. Los ejemplares examinados se asignan con duda al icnogénero Rhizocorallium, ya que, en comparación con las trazas descritas anteriormente, estas no presentan el típico revestimiento alrededor de las estructuras, sus spreiten son diferentes con menos espacios y, aparentemente encimados unos sobre otros, estos se encuentran preservados en epirelieves y sobresalen sobre el sustrato. Lo escaso del material y el mal estado de preservación no permite hacer otro tipo de precisión, sin embargo, las estrías o surcos bien definidos podrían ser parte de un patrón del icnogénero Protovirgularia, ya que en al menos dos de las líneas transversales se observa una forma de chevrón.
También podría tratarse de un registro de Scolicia muy alterado (Figura 6C).

Localidad. Arroyo de las Pulgas, nivel 6 (a 12.5 $m$ de la base en la unidad API-4), Formación Ixtaltepec.

\subsection{ETOLOGÍA Y ORGANISMOS PRODUGTORES DE LAS TRAZAS}

Los icnogéneros presentes en la unidad API-4 de la Formación Ixtaltepec se han relacionado como el registro de diversas actividades realizadas por diferentes organismos:

Cochlichnus. Esta traza se ha considerado como Repichnia (Buatois et al., 1997) y como Pascichnia (Buatois y Mángano, 1995, 2004; Pazos et al., 2007). Como posible productor de este icnogénero, se ha asociado a organismos vermiformes o nemátodos (Tasch, 1968; Metz, 1987; Głuszek, 1995). Igualmente, la icnoespecie C. anguineus se ha relacionado con nemátodos y anélidos (Moussa, 1970; Metz, 1992) o con larvas de insectos (Hakes, 1976).

Gordia. Según la clasificación etológica, Gordia se asigna como Pascichnia (Pazos et al., 2007). Se ha considerado como el producto de la actividad de nemátodos (Häntzschel, 1975).

Lockeia. Según su clasificación etológica se asigna a Cubichnia, pues representa trazas de reposo (Osgood, 1970; Häntzschel, 1975). На sido interpretada como una traza realizada por bivalvos en sustratos suaves (Seilacher y Seilacher, 1994; Mángano et al., 1998).

Planolites. Este icnogénero, según su etología, se ha asignado a Fodinichnia, trazas que reflejan comportamientos de búsqueda de alimento (Paczésna, 1986), a Domichnia, trazas de domicilio y a Pascichnia, trazas de alimentación producidas por organismos micrófagos vágiles (Ekdale, 1985), y también se ha considerado que puede indicar otro tipo de comportamiento superpuesto (Seilacher, 2007). Se ha interpretado como posibles productores a organismos vermiformes (Crimes y Anderson, 1985).

Protovirgularia. Se asigna a Repichnia, por el desplazamiento de bivalvos protobranquios 
vágiles en el interior del sedimento (de Gibert y Domènech, 2008; Carmona et al., 2010).

Rhizocorallium. Esta traza fósil se ha asociado a estructuras que representan la actividad combinada de locomoción y alimentación (Fodinichnia), ya que el organismo se va alimentando a medida que se desplaza sobre el sustrato, formando los surcos (Fraiser y Bottjer, 2009). También ha sido interpretado como Domichnia (Zonneveld et al., 2010). Se ha relacionado con una gran variedad de posibles productores: anélidos (Reis, 1910; Richter, 1927; Schmidt, 1928; Heller, 1930; Hecker, 1965); larvas de insectos (Seilacher, 1955); crustáceos cuando hay presencia de arañazos (Seilacher, 1986; Rodríguez-Tovar y Pérez-Valera, 2008).

\subsection{IGNOASOGIAGIONES}

Los icnotaxones estudiados se encuentran distribuidos en tres icnoasociaciones (AI), ubicadas en diferentes niveles dentro de la unidad API-4 (Figuras 2 y 7):

\subsubsection{ICNOASOCIACIÓN LOCKEIA SILIQUARIA - PROTOVIRGULARIA ISP. (AI-1)}

Se encuentra en la parte inferior de la unidad API-4 (niveles 2 y 3), en una lutita con laminación paralela y ripples de corriente y de interferencia intercaladas. Los icnotaxones presentes son Cochlichnus anguineus, Lockeia siliquaria, Lockeia isp., Gordia isp. y Protovirgularia isp. Representa la parte más diversa y abundante de la unidad, sin embargo, con una baja bioturbación (IB = 1) de acuerdo al modelo de Taylor y Goldring (1993). Los icnotaxones representan estructuras horizontales de Cubichnia (Lockeia siliquaria y Lockeia isp.), Repichnia (Cochlichnus anguineus y Protovirgularia isp.) y Pascichnia (Gordia isp.).

\subsubsection{ICNOASOCIACIÓN LOCKEIA SILIQUARIA - COCHLICHNUS ISP. (AI-2)}

Se encuentra en la parte media de la unidad API-4 (niveles 4 a 7), en capas de lutita arenosa con ripples de interferencia y estratificación tipo flaser hacia la parte superior. Los icnotaxones presentes son Cochlichnus isp., Lockeia siliquaria, Lockeia isp. y
?Rhizocorallium isp. La bioturbación es baja $(\mathrm{IB}=1)$ y la diversidad y abundancia es menor que en AI-1, siendo Lockeia siliquaria la traza mejor representada, mientras que Cochlichnus isp. y ?Rhizocorallium isp. son escasas. Estas trazas son horizontales y representan estructuras de Repichnia (Cochlichnus isp.), Cubichnia (Lockeia siliquaria y Lockeia isp.) y Fodinichnia (? Rhizocorallium isp.).

\subsubsection{ICNOASOCIACIÓN LOCKEIA SILIQUARIA - RHIZOCORALLIUM (AI-3)}

Se encuentra en la parte media-superior de la unidad API-4 (niveles 8 y 9), en capas de lutita, lutita arenosa y arenisca con intercalaciones de ripples de corriente y de interferencia. Los icnotaxones presentes son Lockeia siliquaria, Lockeia isp., Planolites isp. y Rhizocorallium commune. La bioturbación es baja $(\mathrm{IB}=1)$ y la icnodiversidad y abundancia también. El icnotaxón mejor representado es Lockeia siliquaria y en menor abundancia se encuentra Lockeia isp., y son escasos Planolites isp. y Rhizocorallium commune. Las trazas se encuentran horizontalmente, representando estructuras de Cubichnia (Lockeia siliquaria y Lockeia isp.), Fodinichnia o Domichnia (Planolites isp. y Rhizocorallium commune).

\section{Discusión}

Debido a que el concepto de icnofacies se basa en la identificación de ciertas características clave que se comparten por diferentes asociaciones de trazas fósiles durante un amplio rango de edades, bajo condiciones ambientales similares (Crimes, 1977; Seilacher, 1978; Pemberton y Frey, 1984; Buatois y Mángano, 2008, 2011), las icnoasociaciones presentes en la parte inferior y media de la unidad API-4 de la Formación Ixtaltepec corresponden a la icnofacies Cruziana (Seilacher, 1964), según se aplica de forma generalizada (Seilacher, 1964; Frey y Pemberton, 1984; Netto, 1992; Buatois y Mángano, 2008; Desjardins et al., 2012; MacEachern et al., 2012).

La icnofacies Cruziana es una icnofacies marina con una gran variedad de icnogéneros representativos, entre los que se encuentran Gordia, 


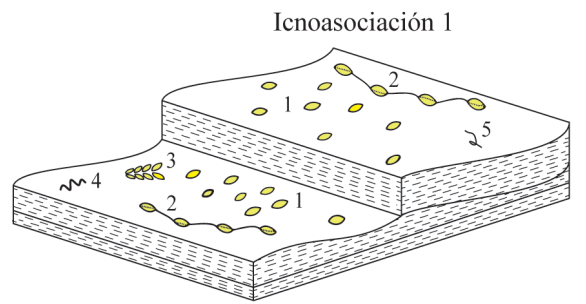

1. Lockeia siliquaria

2. Lockeia isp.

3. Protovirgularia isp.

4. Cochlichnus anguineus

5 ?Gordia isp.

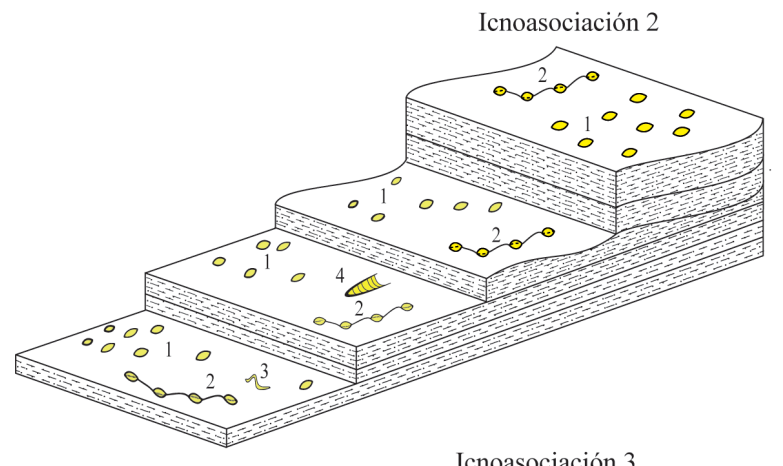

1. Lockeia siliquaria

2. Lockeia isp

3. Cochlichnus isp.

4. Rhizocorallium isp.

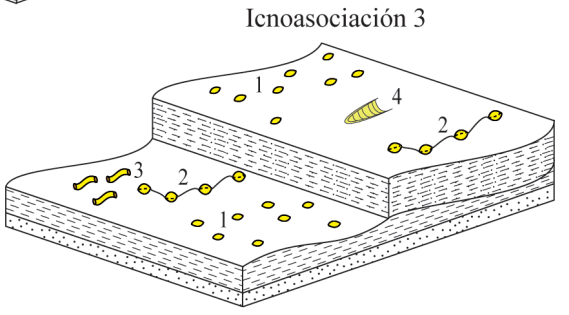

1. Lockeia siliquaria

2. Lockeia isp.

3. Planolites isp.

4. Rhizocorallium commune

Figura 7 Representación de las icnoasociaciones presentes en la unidad API-4 de la Formación Ixtaltepec en su sección tipo "Arroyo de las Pulgas".

Lockeia, Planolites, Protovirgularia y Rhizocorallium (Seilacher, 1964; Pemberton y Frey, 1984; Buatois y Mángano, 2008; Minter et al., 2016), presentes en las icnoasociaciones estudiadas. Otras características que comparten estas icnoasociaciones con la icnofacies Cruziana es la predominancia de trazas horizontales y la amplia gama de categorías etológicas incluyendo trazas de pastoreo (Pascichnia), de locomoción (Repichnia), de descanso (Gubichnia), de alimentación (Fodinichnia) y de habitación (Domichnia), con predominio de trazas producidas por una fauna móvil que indica una alta movilidad de la fauna bentónica. Además, los posibles productores de las trazas estudiadas son nemátodos (Cochlichnus y Gordia), bivalvos (Lockeia y Protovirgularia), anélidos (Cochlichnus y Rhizocorallium), organismos vermiformes (Planolites), crustáceos (Rhizocorallium) y larvas de insectos (Cochlichnus y Rhizocorallium), por lo que están representadas diferentes formas tróficas, predominando los alimentadores de depósito o detrito. De todos ellos, las trazas de bivalvos son las más abundantes y están presentes en las tres icnoasociaciones. En estudios anteriores se ha registrado la presencia de bivalvos del género Phestia en la unidad API-4 (Quiroz-Barroso, 1995; Quiroz-Barroso y Perrillat, 1997), mismos que han sido relacionados como posibles productores de Lockeia (Mángano et al., 1998), por lo que las trazas asignadas al icnogénero Lockeia en esta unidad posiblemente fueron realizadas por especímenes pequeños de este tipo de bivalvos, cuyo tamaño se corresponde con el de las pistas. Por otra parte, Carmona et al. (2010), consideran que el icnogénero Protrovirgularia posee diferentes formas de preservación dependiendo de la consistencia 
del sustrato en donde se desarrolle la estructura, por lo que reconocen cinco morfotipos. La traza estudiada tiene una similitud morfológica con el morfotipo 5, lo que indicaría una consistencia del sustrato en la interfase entre la arena y una delgada capa de fango superpuesta.

La presencia de esta icnofacies implica que en el ambiente de depósito debió existir una acumulación de detrito orgánico en el sedimento, bajo condiciones de energía baja a moderada, lo que permite el desarrollo de detritívoros y la abundancia de trazas horizontales. Por otra parte, se ha relacionado esta icnofacies con una estabilidad ambiental y tasas de sedimentación de baja a moderada, si bien pudo existir sedimentación episódica como ocurre durante las tormentas (Buatois y Mángano, 2008).

La icnofacies Cruziana es una icnofacies de sustrato blando que se caracteriza principalmente por desarrollarse en una plataforma somera, entre el nivel de oleaje normal y el nivel de oleaje de tormenta, pero también se ha relacionado con ambientes someros de energía baja a moderada como lagunas costeras y estuarios (Seilacher, 1964; Frey y Pemberton, 1984; Mángano y Buatois, 2004; Buatois y Mángano, 2008; Desjardins et al., 2012; MacEachern et al., 2012).

La abundancia de trazas de bivalvos detritívoros confirman que el depósito debió ocurrir en la zona intermareal, entre los niveles de marea alta y marea baja, en una planicie de mareas, como también lo indica la presencia de estructuras sedimentarias típicas de estos ambientes: estratificación ondulada y flaser y ripples de corrientes, así como ripples de interferencia (Figura 8). Éstos últimos con diferentes patrones de ripples secundarios caracterizan a las planicies de marea (Chakrabarti, 2005).

En la parte inferior de la unidad API-4, la icnoasociación AI-1 puede asociarse con una planicie de marea alta o fangosa (mud flat), sumergida sólo en el tiempo próximo a la pleamar, cuando apenas hay corrientes. En el repunte de pleamar los fangos en suspensión pueden decantarse y depositarse, produciéndose eventos de colonización múltiple por alimentadores de depósito y pastadores. En esta zona la bioturbación es baja a moderada y la icnodiversidad baja, representando una icnofacies de Cruziana empobrecida (Desjardins et al., 2012). Hacia la parte media de la unidad API-4, las icnoasociaciones AI-2 y AI-3 se presentan en estratos heterolíticos, con estratificación tipo flaser. Lo anterior indicaría un cambio hacia una planicie de marea media o mixta (mixed flat), que se encuentra sumergida y expuesta más o menos igual tiempo y en la que ocurre la sedimentación por carga de fondo de arenas y por decantación de fangos, produciéndose eventos de colonización múltiple por organismos alimentadores de depósito y pastadores. Sin embargo, la bioturbación en estas icnoasociaciones es muy baja, al igual que la icnodiversidad, lo que puede ser indicativo de un cambio a condiciones salobres (Buatois y Mángano, 2008, 2011; Desjardins et al., 2012; Minter et al., 2016), por lo que estas icnoasociaciones representan también una asociación empobrecida de la icnofacies Cruziana.

La intercalación de estratos con trazas fósiles y de ripples de interferencia, indicarían condiciones oscilantes o de estrés ambiental. Este patrón de distribución de los estratos se ha apreciado en otras localidades con la presencia de trazas de locomoción de bivalvos (Lockeia isp.) que se asocian a condiciones de depósito de planicies de marea como en el Miembro Stull Shale del Carbonífero (Pensilvánico Superior) Kansas (Mángano et al., 1998). Las planicies de marea estuarinas salobres o los sistemas de bahía son caracterizados por una baja icnodiversidad marina (Pemberton y Wightman, 1992; Mángano et al., 1998).

En la parte superior de la unidad API-4, que no presenta registro icnológico, están presentes: fósiles corporales de hiolítidos, organismos marinos solitarios, epibentónicos, detritívoros no especializados y prácticamente sésiles, que ocupaban medios de energía baja (Martinell et al., 2009). Los anteriores están asociados a organismos de talla pequeña como: crinoideos, trilobites, bivalvos y braquiópodos, lo cual puede indicar estrés ambiental por posibles cambios de salinidad en el ambiente, condiciones disaeróbicas y un cambio 


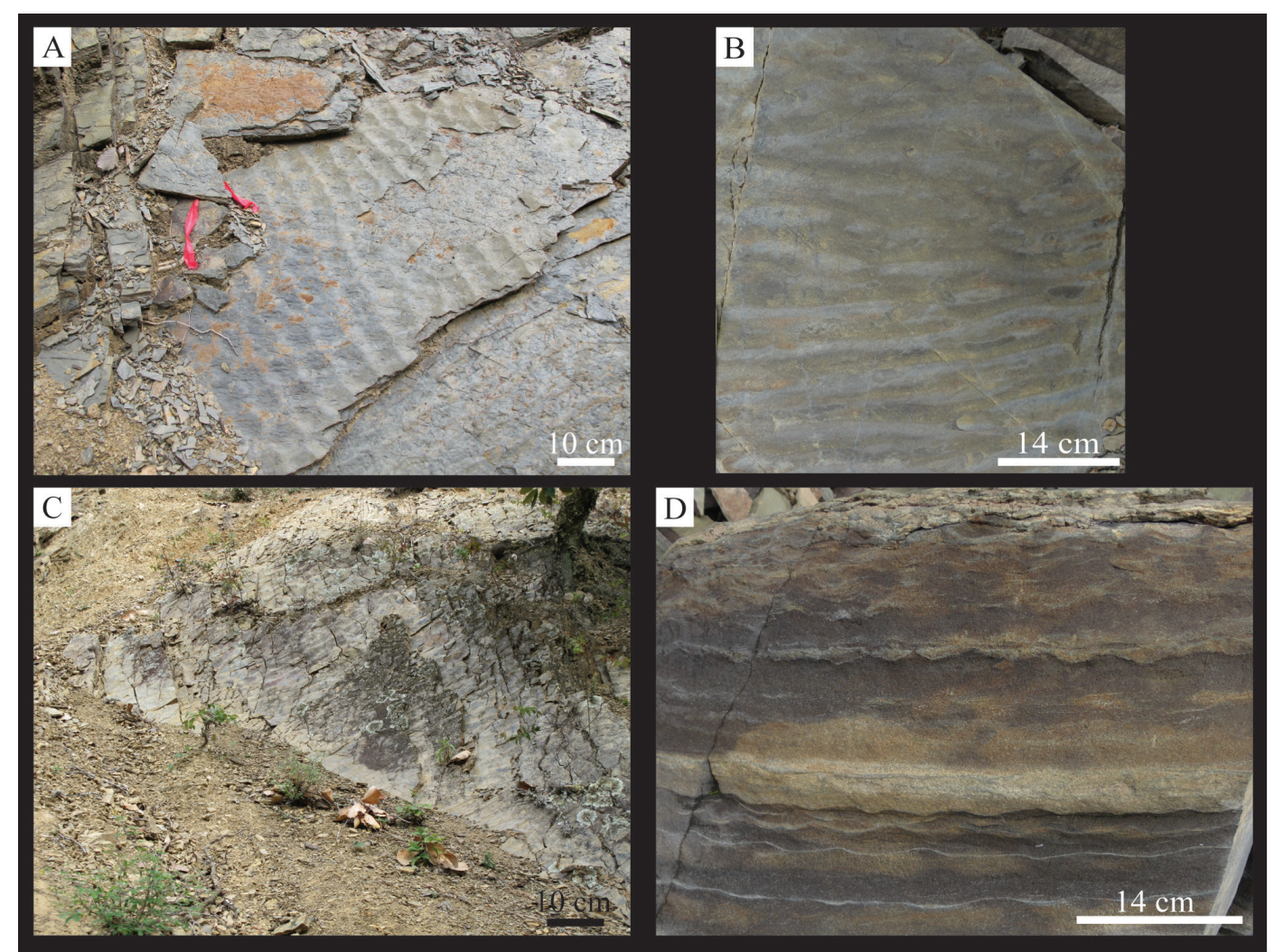

Figura 8 (A) Ripples de interferencia intercaladas con capas con icnófosiles en el nivel 3 de la unidad API-4. (B) Ripples de corriente en la base de la unidad API-4. (C) Ripples de corriente en la parte media de la unidad API-4. (D) Estratificación tipo flaser en la parte media de la unidad API-4.

batimétrico, como se ha referido para algunas localidades del Carbonífero Superior, incluyendo el Mid-Continent de Estados Unidos (Boardman et al., 1984; Kammer et al., 1986).

Esto concuerda con las primeras descripciones ambientales, en donde Pantoja-Alor (1970) menciona que la abundancia de sedimentos arcillosos con intercalaciones de arenisca en la Formación Ixtaltepec es un indicio del hundimiento paulatino de la cuenca, con periodos de máximo acarreo de materiales o cambios en la línea de costa. En este contexto, la parte inferior y media de la unidad API-4 indica una etapa de somerización cuya evidencia son los icnofósiles y la presencia de plantas fósiles, aunque escasas, asociados a ripples de interferencia y estratificación tipo flaser (Figura 9).

\section{Conclusiones}

El registro icnológico que está presente en la parte inferior y media de la unidad API-4 de la Formación Ixtaltepec indica que el depósito ocurrió en un ambiente de planicie de marea, dentro de la zona intermareal. Las diferentes icnoasociaciones contienen una predominancia de pistas de bivalvos detritívoros y se relacionan con mudflat-mixed flat, con una baja bioturbación del sedimento, lo que indica una icnofacies Cruziana empobrecida.

El estudio icnológico permite conocer las condiciones en las que se produjo el depósito de esta unidad y así complementar el conocimiento de los cambios ambientales que ocurrieron en el área 


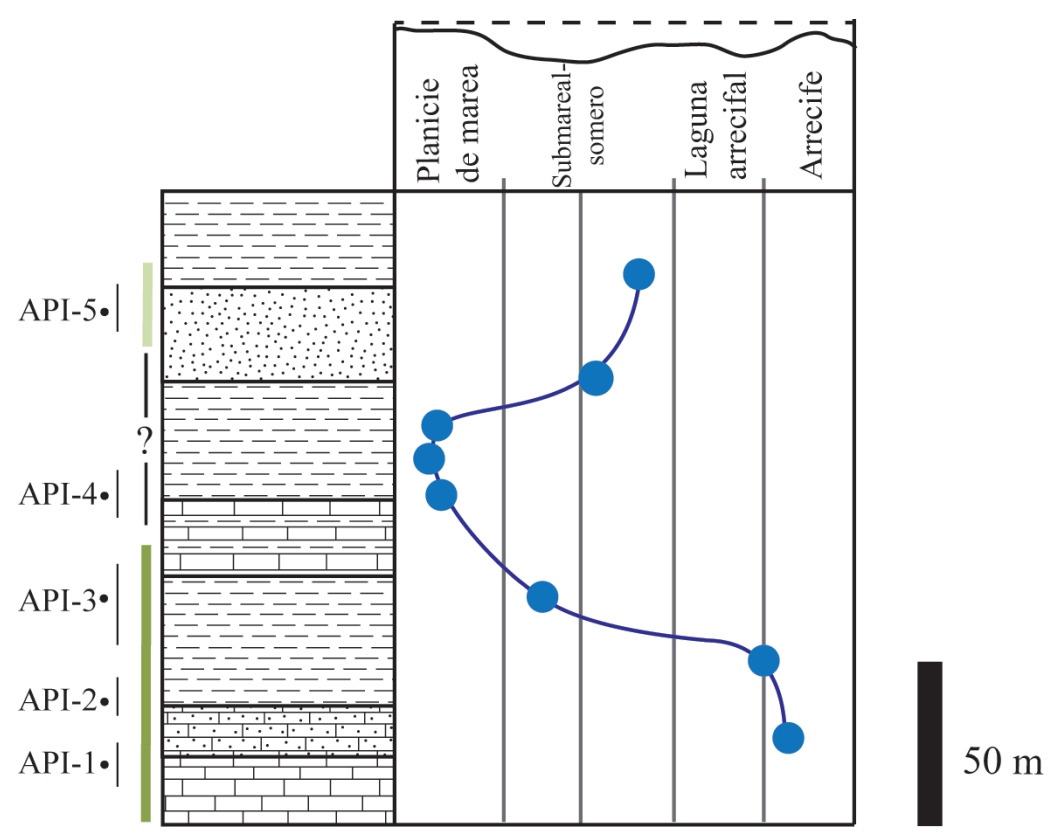

Figura 9 Paleoambientes inferidos en las unidades API-1 a API-3, parte superior de la unidad API-4 y API-5, a partir del análisis tafonómico y de los fósiles corporales. La interpretación paleoambiental de la base y parte media de la unidad API-4 se infiere con el estudio icnológico.

de estudio. La interpretación que se hace de un ambiente de planicie de marea relacionado con esta unidad indica una etapa de somerización cuya evidencia son los mismos icnofósiles y la presencia de escasos restos de plantas fósiles, asociados a ripples de corriente y de interferencia y a una estratificación tipo flaser.

Por otra parte, la mayoría de los icnofósiles estudiados proveen información de la existencia de organismos que por ser de cuerpo blando no han dejado un registro corporal, lo que provee información adicional para el conocimiento de la diversidad biológica que existió en el área de estudio durante el Serpukhoviano-Bashkiriano.

\section{Agradecimientos}

Las autoras agradecen a Francisco Vega-Vera y a dos revisores anónimos la cuidadosa revisión del manuscrito, todos sus comentarios contribuyeron a mejorarlo de manera sustancial. A Daniel Navarro Santillán por su ayuda técnica en el trabajo de campo y a Héctor Hernández Campos por la elaboración de las fotografías de los icnofósiles. $\mathrm{Al}$ Posgrado en Ciencias Biológicas de la Universidad Nacional Autónoma de México y al Consejo Nacional de Ciencia y Tecnología (CONACYT), por la beca otorgada durante los estudios de maestría de la primera autora. A la Dirección General de Asuntos del Personal Académico (DGAPA) de la Universidad Nacional Autónoma de México, por el apoyo económico brindado a través del proyecto PAPIIT IN215013-2.

\section{Referencias}

Balistieri, P.R.M.N., Netto, R.G., Lavina, E.L.G., 2002, Ichnofauna from the Upper Carboniferous-Lower Permian rhythmites from Mafra, Santa Catarina State, Brazil: Ichnotaxonomy: Revista Brasileira de Paleontologia, 4, 13-26.

Bandel, K., 1967, Trace fossils from two Upper Pennsylvanian sandstones in Kansas: The University of Kansas Paleontological Contributions, 18, 1-13. 
Billings, E., 1862, New species of fossils from different parts of the Lower, Middle and Upper Silurian rocks of Canada: Geological Survey of Canada, Palaeozoic Fossils, 1, 96-168.

Boardman , D.R.II, Mapes, R.H., Yancey, T.E., Malinky, J.M., 1984, A new model for the depth-related allogenic community succession within North American Pennsylvanian cyclothems and implications on the black shale problem, en Hyne, N.J. (ed.), Limestones of the Mid-Continent: Tulsa, Oklahoma, E.U.A., Tulsa Geological Society Special Publication, 2, 141-182.

Bromley, R.G., 1996, Trace fossils: biology, taphonomy and applications: Londres, Reino Unido, Chapman \& Hall, 361 p.

Buatois, L.A., Mángano, M.G., 1995, The paleoenvironmental and paleoecological significance of the lacustrine Mermia ichnofacies: An archetypical subaqueous nonmarine trace fossil assemblage: Ichnos, 4(2), 151-161.

Buatois, L.A., Mángano, M.G., 2004, Animalsubstrate interactions in freshwater environments: applications of ichnology in facies and sequence stratigraphic analysis of fluvio-lacustrine successions, en McIlroy, D. (ed.), The Application of Ichnology to Palaeoenvironmental and Stratigraphic Analysis: Londres, Reino Unido, Geological Society Special Publication, 228, 311-333.

Buatois, L.A., Mángano, M.G., 2007, Invertebrate ichnology of continental freshwater environments, en Miller, W.III (ed.), Trace Fossils: Concepts, Problems, Prospects: Oxford, Reino Unido, Elsevier, 285-323.

Buatois, L.A., Mángano, M.G., 2008, Trazas fósiles de invertebrados, en Camacho, H.H., Longobucco, M. (eds.), Los Invertebrados Fósiles: Buenos Aires, Argentina, Fundación de Historia Natural Félix de Azara, Vázquez Mazzini, 751-785.

Buatois, L.A., Mángano, M.G., 2011, Ichnology: Organism-Substrate Interactions in Space and Time: Cambridge, Reino Unido, Cambridge University Press, 358 p.

Buatois, L.A., Jalfin, G., Aceñolaza, F.G., 1997,

Permian Nonmarine Invertebrate Trace Fossils from Southern Patagonia, Argentina: Ichnologic Signatures of Substrate Consolidation and Colonization Sequences: Journal of Paleontology, 71(2), 324-336.

Buatois, L.A., Mángano, M.G., Maples, G.G., Lanier, W.P., 1998, Ichnology of an Upper Carboniferous fluvio-estuarine paleovalley: The Tonganoxie Sandstone, Buildex Quarry, eastern Kansas, USA: Journal of Paleontology, 72(1), 152-180.

Carmona, N.B., Mángano, M.G., Buatois, L.A., Ponce, J.J., 2010, Taphonomy and paleoecology of the bivalve trace fossil Protovirgularia in deltaic heterolithic facies of the Miocene Chenque Formation, Patagonia, Argentina: Journal of Paleontology, 84, 730-738.

Castillo-Espinoza, K.M., 2013, Sistemática de braquiópodos, cefalópodos y crinoideos del misisípico medio de la Formación Santiago, Santiago Ixtaltepec, Oaxaca: Distrito Federal, México, Universidad Nacional Autónoma de México, Tesis de Maestría, 112 p.

Chakrabarti, A., 2005, Sedimentary structures of tidal flats: A journey from coast to inner estuarine region of eastern India: Journal of Earth System Science, 114(3), 353-368.

Crimes, T.P., 1977, Trace fossils of an Eocene deep-sea sand fan, northern Spain, en Crimes, T.P., Harper, J.C. (eds)., Trace fossils 2: Geological Journal Special Issue, 9, 71-90. Crimes, T.P., Anderson, M.M., 1985, Trace fossils from Late Precambrian-Early Cambrian strata of southeastern Newfoundland (Canada): temporal and environmental implications: Journal of Paleontology, 59(2), 310-343.

Crimes, T.P., Fedonkin, M.A., 1994, Evolution and dispersal of deepsea traces: Palaios, 9, 74-83. 
de Gibert, J.M., Domènech, R., 2008, Trazas fósiles de nuculoideos (Protovirgularia) del Mioceno marino de la Guenca del VallèsPenedès: Revista Española de Paleontología, 23(2), 129-138.

de Gibert, J.M., Martinell, J., 1998, El modelo de icnofacies, 30 años después: Revista Española de Paleontología, 13(2), 167-174.

Desjardins, P.R., Buatois, L.A., Mángano, M.G., 2012, Tidal Flats and Subtidal Sand Bodies, en Knaust, D.K., Bromley, R.G. (eds.), Trace Fossils as Indicators of Sedimentary Environments: Developments in Sedimentology, 64, 529-561.

Ekdale, A.A., 1985, Paleoecology of the marine endobenthos: Palaeogeography, Palaeoclimatology, Palaeoecology, 50, 63-81.

Emmons, E., 1844, The Taconic System; Based on Observations in New-York, Massachusetts, Maine, Vermont and Rhode-Island: Albany, Nueva York, Carroll and Cook, 68 p.

Fillion, D., Pickerill, R.K., 1990, Ichnology of the Upper Cambrian? to Lower Ordovician Bell Island and Wabana groups of eastern Newfoundland, Canada: Palaeontographica Canadiana, 7, 1-119.

Fraiser, M.L., Bottjer, D.J., 2009, Opportunistic behaviour of invertebrate marine tracemakers during the Early Triassic aftermath of the end-Permian mass extinction: Australian Journal of Earth Sciences 56, 841-857.

Frey, R.W., Pemberton, S.G., 1984, Trace Fossils Facies Models, en Walker, R.G. (ed.), Facies Models: Newfoundland, Canada, Geoscience Canada Reprint Series, 1, 189-207.

Fries, C., Schmitter, E., Damon, P.E., Livingstone, D.E., 1962, Rocas Precámbricas de edad Grenvilliana de la parte central de Oaxaca en el Sur de México: Boletín del Instituto de Geología, Universidad Nacional Autónoma de México, 64, 45-53.

Garcia-Ramos, J.C., 1984, Estudio de las trazas fósiles (Icnofauna) del campo de Gibraltar
(Provincia de Cádiz): Madrid, España, Instituto Geológico y Minero de España, Informe interno Magna, 48, 1-97.

Głuszek, A., 1995, Invertebrate trace fossils in the continental deposits of an Upper Carboniferous coal-bearing succession, Upper Silesia, Poland: Studia Geologica Polonica, 108, 171-202.

González-Mora, S., 2013, Briozoarios del orden Fenestrida de la Formación Ixtaltepec, Carbonífero del Municipio de Nochixtlán, Oaxaca: Distrito Federal, México, Universidad Nacional Autónoma de México, Tesis de Licenciatura, $41 \mathrm{p}$.

González-Mora, S., 2017, Sistemática de los briozoarios carboníferos de Nochixtlán, Oaxaca: modelado geométrico y determinación de paleoambientes: Cuidad de México, México, Universidad Nacional Autónoma de México, Tesis de Maestría, $101 \mathrm{p}$

González-Mora, S., Sour-Tovar, F., 2014, Briozoarios del Orden Fenestrida, Pensilvánico de la Formación Ixtaltepec, Municipio de Nochixtlán, Oaxaca; consideraciones paleoambientales: Boletín de la Sociedad Geológica Mexicana, 66(3), 471-482.

Gümbel, K.W.von, 1879, Geognostische Beschreibung de Fichtelgebirges mit dem Frankenwalde und dem westlichen Vorlande: Gotha, Alemania, Justus Perthes, 3, 698 p.

Hakes, W.G., 1976, Trace fossils and depositional environment of four clastic units, Upper Pennsylvanian Megacyclotherms, Northeast Kansas: The University of Kansas Paleontological Contributions, 63, 1-60.

Han, Y., Pickerill, R.K., 1995, Taxonomic review of the ichnogenus Helminthopsis Heer 1877 with a statistical analysis of selected ichnospecies: Ichnos, 4, 83-118. 
Häntzschel, W., 1975, Trace fossils and problematica, en Moore, R.C. (ed.), Treatise on Invertebrate Paleontology, Part W, Miscellanea, Supplement 1: Lawrence, E.U.A., Geological Society of America, University of Kansas Press, 1-269.

Hecker, R.F., 1965, Introduction to Paleoecology: Nueva York, E.U.A., American Elsevier Publishing Company, 166 p.

Heller, F., 1930, Geologische Untersuchungen im Bereiche des Fränkischen Grundgipses: Abhandlungen der Naturhistorischen Gesellschaft Nürnberg, 23, 45-114.

Hernández-Ocaña, M.I., 2016, Estudio icnológico y tafonómico de la Formación Ixtaltepec, Carbonífero de Nochixtlán, Oaxaca: Ciudad de México, México, Universidad Nacional Autónoma de México, Tesis de Maestría, $111 \mathrm{p}$.

Hitchcock, E., 1858, Ichnology of New England; A report on the sandstone of the Connecticut Valley, especially its fossil footmarks: Boston, E.U.A., William White, 220 p.

James, U.P., 1879, Description of new species of fossils and remarks on some others, from the Lower and Upper Silurian rocks of Ohio: The Paleontologist, 3, 17-24.

Kammer, T.W., Brett, C.E., Boardman, D.R.II, Mapes, R.H., 1986, Ecologic stability of the dysaerobic biofacies during the Late Paleozoic: Lethaia, 19(2), 109-121.

Keighley, D.G., Pickerill, R.K., 1995, Commentary: the ichnotaxa Palaeophycus and Planolites, historical perspective and recommendations: Ichnos, 3, 301-309.

Keighley, D.G., Pickerill, R.K., 1997, Systematic ichnology of the Mabou and Cumberland groups (Carboniferous) of western Cape Breton Island, eastern Canada, 1: burrows, pits, trails, and coprolites: Atlantic Geology, 33(3), 181-215.

Knaust, D., 2013, The ichnogenus Rhizocorallium: Classification, trace makers, palaeoenvironments and evolution: EarthScience Reviews, 126, 1-47.

M'Coy, F., 1850, On some new genera and species of Silurian Radiata in the collection of the University of Cambridge: Annals and Magazine of Natural History, Series 2, 6, 270-290.

MacEachern, J.A., Gingras, M.K., Bann, K.L, Dafoe, L.T., Pemberton, S.G., 2007, Applications of ichnology to highresolution genetic stratigraphic paradigms, en MacEachern, J.A., Bann, K.L., Gingras, M.K., Pemberton, S.G. (eds.), Applied Ichnology: Tulsa, Oklahoma, E.U.A., Society of Economic Paleontologists and Mineralogists, Short Course Notes, 52, 95-129.

MacEachern, J.A., Bann, K.L., Gingras, M.K., Zonneveld,J.-P., Dashtgard, S.E., Pemberton, S.G., 2012, The Ichnofacies Paradigm, en Knaust, D., Bromley, R.G. (eds.), Trace Fossils as Indicators of Sedimentary Environments: Developments in Sedimentology, 64, 103-138.

Mángano, M.G., Buatois, L.A., 2004, Ichnology of Carboniferous tide-influenced environments and tidal flat variability in the North American Midcontinent, en McIlroy, D. (ed.), The Application of Ichnology to Palaeoenvironmental and Stratigraphic Analysis: Londres, Reino Unido, Geological Society Special Publication, 228, 157-178.

Mángano, M.G., Buatois, L.A., West, R.R., Maples, C.G., 1998, Contrasting behavioral and feeding strategies recorded by tidalflat bivalve trace fossils from the Upper Carboniferous of eastern Kansas: Palaios, 13, 335-351.

Martinell, J., Checa, A., Domènech, R., Gili, C., Olóriz, F., Rodríguez-Tovar, FJ., 2009, Moluscos, en Martínez-Chacón, M.L., Rivas, P. (eds.), Paleontología de Invertebrados: Oviedo, Asturias, España, Universidad de Oviedo, 227-376. 
McIlroy, D., 2004, The Application of Ichnology to Palaeoenvironmental and Stratigraphic Analysis: Londres, Reino Unido, Geological Society of London Special Publication, 228, $490 \mathrm{p}$.

Metz, R., 1987, Sinusoidal trail formed by a recent biting midge (family Ceratopogonidae): Trace fossil implications: Journal of Paleontology, 61(2), 312-314.

Metz, R., 1992, Trace fossils from the Lower Jurassic nonmarine Towaco Formation, New Jersey: Northeastern Geology Incorporating Northeastern Environmental Science, 14, 29-34.

Minter, N.J., Buatois, L.A., Mángano, M.G., 2016, The Conceptual and Methodological Tools of Ichnology, en Mángano, M.G., Buatois, L.A., (eds.), The Trace-Fossil Record of Major Evolutionary Events: Precambrian and Paleozoic: Dordrecht, Países Bajos, Springer, Topics in Geobiology, 39, 1-26.

Morón, A., Perrilliat, M.G., 1988, Una nueva especie del género Griffithides Portlock (Arthropoda: Trilobita) del Paleozoico superior de Oaxaca: Revista del Instituto de Geología, Universidad Nacional Autónoma de México, 7, 67-70.

Moussa, M.T., 1970, Nematode fossil trails from the Green River Formation (Eocene) in the Uinta Basin, Utah: Journal of Paleontology, 44, 304-307.

Navarro-Santillán, D., Sour-Tovar, F., CentenoGarcía, E., 2002, Lower Mississippian (Osagean) brachiopods from the Santiago Formation, Oaxaca, Mexico: stratigraphic and tectonic implications: Journal of South American Earth Sciences, 15, 327-336.

Netto, R.G., 1992, Paleoecology of ichnocoenoses in the Cruziana Ichnofacies, Serrinha Member, Rio do Rasto Formation, (Upper Permian) from the Parana Basin, Rio Grande do Sul, Brazil: Acta Geologica Leopoldensia, $36,67-82$.

Nicholson, H.A., 1873, Contribution to the study of errant annelids of the Older Paleozoic rocks: Proceedings of the Royal Society of London, 21, 288-290.

Olivero, D., 2003, EarlyJurassic to Late Cretaceous evolution of Zoophycos in the French Subalpine Basin (southeastern France): Palaeogeography, Palaeoeclimatology, Palaeoecology, 192, 59-78.

Olivero, D., Gaillard, G., 1996, Palaeoecology of Jurassic Zoophycos from South-Eastern France: Ichnos, 4, 249-260.

Ortiz-Lozano, J.A., 1996, Consideraciones paleoambientales y morfológicas de las colonias de Briozoarios Pensilvánicos de la Formación Ixtaltepec en la región de Nochixtlán, Oaxaca: Distrito Federal, México, Universidad Nacional Autónoma de México, Tesis de Licenciatura, 40 p.

Osgood, R.G.jr., 1970, Trace fossils of the Cincinnati area: Palaeontographica Americana, 6, 281- 438.

Paczésna, J., 1986, Upper Vendian and Lower Cambrian ichnocoenoses of Lublin region (Poland): Biuletyn Instytutu Geologicznego, 355, 31-47.

Pantoja-Alor, J., 1970, Rocas sedimentarias paleozoicas de la región centro-septentrional de Oaxaca, en Segura, L.R., Rodríguez, T.R. (eds.), Libro guía de la excursión MéxicoOaxaca: Distrito Federal, México, Sociedad Geológica Mexicana, 67-84.

Pantoja-Alor, J., Robison, R.A., 1967, Paleozoic sedimentary rocks in Oaxaca, Mexico: Science, 157, 1033-1035.

Pazos, P.J., Di Pasquo, M., Rodríguez-Amenabar, C., 2007, Trace Fossils of the Glacial to Postglacial Transition in the El Imperial Formation (Upper Carboniferous), San Rafael Basin, Argentina, en Bromley, R.G., Buatois, L.A., Mángano, M.G., Genise, J.F., Melchor, R.N. (eds.), Sediment-Organism Interactions: A Multifaceted Ichnology: Tulsa, Oklahoma, E.U.A., Society of Economic Paleontologists and Mineralogists, Special Publication, 88, 137-147. 
Pemberton, S.G., Frey, R.W., 1982, Trace fossils nomenclature and the Planolites-Palaeophycus Dilemma: Journal of Paleontology, 56(4), 843-881.

Pemberton, S.G., Frey, R.W., 1984, Ichnology of Storm-Influenced Shallow Marine Sequence: Cardium Formation (Upper Cretaceous) at Seebe, Alberta, en Stott, D.F., Glass, D.J. (eds.), The Mesozoic of Middle North America: A Selection of Papers from the Symposium on the Mesozoic of Middle North America, Calgary, Alberta, Canada: Calgary, Canada, Canadian Society of Petroleum Geologists, 9, 281-304.

Pemberton, S.G., Wightman, D.M., 1992, Ichnological characteristics of brackish water deposits, en Pemberton, S.G. (ed.), Applications of Ichnology to Petroleum Exploration: A Core Workshop: Tulsa, Oklahoma, E.U.A., Society of Economic Paleontologists and Mineralogists, Core Workshop Notes, 17, 141-167.

Pemberton, S.G., MacEachern, J.A., Gingras, M.K., Zhang, J., 2000, Significance of ichnofossils to genetic stratigraphy: Science in China Series-D, Earth Sciences, 43(5), 541-560.

Peña-Salinas, M.E., 2014, Análisis Sistemático de los Corales Rugosos de la Formación Ixtaltepec, Carbonífero del Municipio de Nochixtlán, Oaxaca: Distrito Federal, México, Universidad Nacional Autónoma de México, Tesis de Licenciatura, 66 p.

Pickerill, R.K., Peel, J.S., 1991, Gordia nodosa isp. nov. and other traces from the Cass Fjord Formation (Cambrian) of North Greenland: Rapport Grønlands Geologiske Undersøgelse, 150, 15-28.

Quiroz-Barroso, S.A., 1995, Bivalvos del Carbonífero de Nochixtlán, Oaxaca: Distrito Federal, México, Universidad Nacional Autónoma de México, Tesis doctoral, 86 p. Quiroz-Barroso, S.A., Perrilliat, M.C., 1997, Pennsylvanian Nuculoids (Bivalvia) from the Ixtaltepec Formation, Oaxaca, Mexico:
Journal of Paleontology, 71(3), 400-407.

Quiroz-Barroso, S.A., Perrilliat, M.C., 1998, Pennsylvanian bivalves from the Ixtaltepec Formation, Mexico: Journal of Paleontology, 72(6), 1011-1024.

Quiroz-Barroso, S.A., Sour-Tovar, F., 1995,

Nuevo registro de ofiuroideo (Ophiurinidae) para el Pensilvánico de América del Norte, proveniente de la Formación Ixtaltepec, Oaxaca (resumen), en Memoria del V Congreso Nacional de Paleontología: Mexico, Sociedad Mexicana de Paleontología, 31. Quiroz-Barroso, S.A., Pojeta, J.jr., Sour-Tovar, F., Morales-Soto, S., 2000, Pseudomulceodens: a Mississippian Rostroconch from Mexico: Journal of Paleontology, 74, 1184-1 186.

Reis, O.M., 1910, Beobachtungen über Schichtenfolge und Gesteinsausbildungen in der fränkischen Unteren und Mittleren Trias: Geognostische Jahreshefte, 22, 1-285. Richter, R., 1853, Thüringische Graptolithen: Zeitschrift der Deutschen Geologischen Gesellschaft, 5, 439-464.

Richter, R., 1927, Die fossilen Fährten und Bauten der Würmer, ein Überblick über ihre biologischen Grundformen und deren geologische Bedeutung: Paläontologische Zeitschrift, 9, 193-235.

Richter, R., 1937, Marken und Spuren aus allen Zeiten, I-II: Senckenbergiana, 19, 150-169.

Richter, R., 1941, Marken und Spuren im Hunsrückschiefer. III. Fährten als Zeugnisse des Lebens auf dem Meeresgrunde: Senckenbergiana lethaea, 23, 218-260.

Rodríguez-Tovar, F.J., Pérez-Valera, F., 2008, Trace fossil Rhizocorallium from the Middle Triassic of the Betic Cordillera, Southern Spain: characterization and environmental implications: Palaios, 23, 78-86.

Schlirf, M., Uchman, A., Kümmel, M., 2001, Upper Triassic (Keuper) non-marine trace fossils from the Haßberge area (Franconia, south-eastern Germany): Paläontologische Zeitschrift, 75, 71-96. 
Schmid, E.E., 1876, Der Muschelkalk des östlichen Thüringen: Jena, Alemania, Fromann, 20 p. Schmidt, M. 1928. Die Lebewelt unserer Trias: Öhringen, Alemania, Hohenlohe'sche Buchhandlung Ferdinand Rau, 461 p.

Seilacher, A., 1953, Studien zur Palichnologie. II. Die fossilien Ruhespuren (Cubichnia): Neues Jahrbuch für Geologie und Paläontologie, Abhandlungen, 98, 87-124.

Seilacher, A., 1955, Spuren und Fazies im Unterkambrium. Beiträge zur Kenntnis des Kambriums in der Salt Range (Pakistan), en Schindewolf, O.H., Seilacher, A. (eds.), Akademie der Wissenschaften und der Literatur zu Mainz, mathematischnaturwissenschaftliche Klasse: Abhandlungen, 10, 373-399.

Seilacher, A., 1964, Announcement: Sedimentological classification and nomenclature of trace fossils: Sedimentology, 3, 253-256.

Seilacher, A., 1967, Bathymetry of trace fossils: Marine Geology, 5, 413-428.

Seilacher, A., 1978, Use of trace fossil assemblages for recognizing depositional environments, en Basan, P.B. (ed.), Trace fossil concepts: Tulsa, Oklahoma, E.U.A., Society of Economic Paleontologists \& Mineralogists, Short Course, 5, 167-181.

Seilacher, A., 1986, Evolution of behavior as expressed in marine trace fossils, en Nitecki, M.H., Kitchell, J.A., (eds.), Evolution of Animal Behavior: Paleontological and Field Approaches: Nueva York, E.U.A., Oxford University Press, 62-87 p.

Seilacher, A., 2007, Trace Fossil Analysis: Heilderberg, Alemania, Springer-Verlag, 226 p.

Seilacher, A., Seilacher, E., 1994, Bivalvian trace fossils: A lesson from actuopaleontology: Courier For-schungsinstitut Senckenberg, $169,5-15$.
Silva-Pineda, A., 1970, Fructificación de pteridosperma en el Pensilvánico de Oaxaca (resumen), en Primera Convención Nacional de la Sociedad Geológica Mexicana: México, Sociedad Geológica Mexicana, 85-86 p.

Solari, L.A., Keppie, J.D., Ortega-Gutiérrez, F., Cameron, K.L., Lopez, R., Hames, W.E., 2003, 990 and 1100 Ma Grenvillian tectonothermal events in the northern Oaxacan Complex, southern Mexico: roots of an orogen: Tectonophysics, 365, 257-282.

Sour-Tovar, F., 1994, Braquiópodos pensilvánicos del área de Santiago Ixtaltepec, Municipio de Nochixtlán, Oaxaca: Distrito Federal, México, Universidad Nacional Autónoma de México, Tesis de Maestría, 55 p.

Sour-Tovar, F., Buitrón-Sánchez, B.E., 1987, Los graptolitos del Tremadociano de Ixtaltepec, Oaxaca. Consideraciones sobre el límite Cámbrico-Ordovícico de la región: Revista de la Sociedad Mexicana de Paleontología, $1,380-395$.

Sour-Tovar, F., Martínez-Chacón, M.L., 2004, Braquiópodos chonetoideos del Carbonífero de México: Revista Española de Paleontología, 19, 125-138.

Sour-Tovar, F., Quiroz-Barroso, S.A., 1989, Braquiópodos pensilvánicos (Strophomenida) de la Formación Ixtaltepec, Santiago Ixtaltepec, Oaxaca: Revista de la Sociedad Mexicana de Paleontología, 2, 5-17.

Stanley, D.C.A., Pickerill, R.K., 1998, Systematic ichnology of the Late Ordovician Georgian Bay Formation of Southern Ontario, Eastern Canada: Royal Ontario Museum Life Sciences Contributions, 162, 1-56.

Tasch, P., 1968, A Permian trace fossil from the Antarctic Ohio range: Transactions of the Kansas Academy of Sciences, 71, 33-37.

Taylor, A.M., Goldring, R., 1993, Description and analysis of bioturbation and ichnofabric: Journal of the Geological Society, 150, 141-148. 
Torres-Martínez, M.A., 2014, Braquiópodos Carboníferos del área de Santiago Ixtaltepec, Oaxaca: Implicaciones Paleoambientales, Estratigráficas y Paleobiogeográficas: Distrito Federal, México, Universidad Nacional Autónoma de México, Tesis doctoral, 191 p.

Torres-Martínez, M.A., Sour-Tovar, F., 2012,

Nuevos braquiópodos prodúctidos (Rhynchonelliformea, Strophomenata) del Carbonífero de la región de Nochixtlán, Oaxaca: Revista Mexicana de Ciencias Geológicas, 29, 696-712.

Torres-Martínez, M.A., Sour-Tovar, F., 2016a, Braquiópodos discínidos (Lingulida, Discinoidea) de la Formación Ixtaltepec, Carbonífero del área de Santiago Ixtaltepec, Oaxaca: Boletín de la Sociedad Geológica Mexicana, 68(2), 313-321.

Torres-Martínez, M.A., Sour-Tovar, F., 2016b,

New productide brachiopods (Productoidea) from the Carboniferous of Ixtaltepec Formation, Oaxaca, Mexico: Journal of Paleontology, 90(3), 418-432.

Villanueva-Olea, R., 2011, Crinoideos del Carbonífero de la región de Nochixtlán, Oaxaca: Distrito Federal, México, Universidad Nacional Autónoma de México, Tesis de Maestría, 85 p.
Villanueva-Olea, R., Sour-Tovar F., 2014, A new genus and four new species of cladid crinoids from the Carboniferous of Oaxaca State, Mexico: Journal of Systematic Paleontology, 13(7), 527-542.

Villanueva-Olea, R., Castillo-Espinoza, K.M., Sour-Tovar, F., Quiroz-Barroso, S.A., Buitrón-Sánchez, B.E., 2011, Placas columnares de crinoides del Carbonífero de la región de Santiago Ixtaltepec, Municipio de Nochixtlán, Oaxaca; consideraciones estratigráficas y paleobiogeograficas: Boletín de la Sociedad Geológica Mexicana, 63(3), 429-443.

Volk, M., 1961, Protovirgularia nereitarum (Reinhard Richter), eine Lebensspur aus dem Devon Thüringen: Senckenbergiana Lethaea, 42, 69-75.

Zenker, J.C., 1836, Historisch-topographisches Taschenbuch von Jena und seiner Umgebung: Jena, Alemania, Friedrich Frommann, 338 p.

Zonneveld, J.P., Gingras, M.K., Beatty, T.W., 2010, Diverse ichnofossil assemblages following the P-T mass extinction, Lower Triassic, Alberta and British Columbia, Canada: evidence for shallow marine refugia on the northwestern coast of Pangaea: Palaios, 25, 368-392. 